\title{
Mice harboring the FXN I151F pathological point mutation present decreased frataxin levels, a Friedreich ataxia-like phenotype, and mitochondrial alterations
}

\author{
Marta Medina-Carbonero ${ }^{1} \cdot$ Arabela Sanz-Alcázar $^{1}$ • Elena Britti ${ }^{1} \cdot$ Fabien Delaspre $^{1} \cdot{\text { Elisa } \text { Cabiscol }^{1} \text { · Joaquim Ros }}^{1}$. \\ Jordi Tamarit ${ }^{1}$ iD
}

Received: 25 May 2021 / Revised: 21 November 2021 / Accepted: 16 December 2021 / Published online: 17 January 2022

(c) The Author(s) 2022

\begin{abstract}
Friedreich Ataxia (FA) is a rare neuro-cardiodegenerative disease caused by mutations in the frataxin $(F X N)$ gene. The most prevalent mutation is a GAA expansion in the first intron of the gene causing decreased frataxin expression. Some patients present the GAA expansion in one allele and a missense mutation in the other allele. One of these mutations, FXNI154F, was reported to result in decreased content of mature frataxin and increased presence of an insoluble intermediate proteoform in cellular models. By introducing this mutation into the murine Fxn gene (I151F, equivalent to human I154F) we have now analyzed the consequences of this pathological point mutation in vivo. We have observed that FXN ${ }^{\mathrm{I} 151 \mathrm{~F}}$ homozygous mice present low frataxin levels in all tissues, with no evidence of insoluble proteoforms. Moreover, they display neurological deficits resembling those observed in FA patients. Biochemical analysis of heart, cerebrum and cerebellum have revealed decreased content of components from OXPHOS complexes I and II, decreased aconitase activity, and alterations in antioxidant defenses. These mitochondrial alterations are more marked in the nervous system than in heart, precede the appearance of neurological symptoms, and are similar to those observed in other FA models. We conclude that the primary pathological mechanism underlying the I151F mutation is frataxin deficiency, like in patients carrying GAA expansions. Therefore, patients carrying the I154F mutation would benefit from frataxin replacement therapies. Furthermore, our results also show that the $\mathrm{FXN}^{\mathrm{I} 151 \mathrm{~F}}$ mouse is an excellent tool for analyzing tissue-specific consequences of frataxin deficiency and for testing new therapies.
\end{abstract}

Keywords Friedreich Ataxia $\cdot$ Iron-sulfur $\cdot$ Mitochondria $\cdot$ Oxidative stress $\cdot$ OXPHOS

\section{Introduction}

Friedreich Ataxia (FA) is a rare, inherited recessive disease first described by Nikolaus Friedreich, a German pathologist, in 1863. FA is characterized by progressive gait and limb ataxia with associated limb muscle weakness, absent lower limb reflexes, extensor plantar responses, dysarthria, and decreased vibratory sense and proprioception [1]. Most FA patients also present hypertrophic cardiomyopathy, cardiac dysfunction being the leading cause of death [2]. The

Jordi Tamarit

Jordi.tamarit@udl.cat

Dept. Ciències Mèdiques Bàsiques, Fac. Medicina, IRBLleida, Universitat de Lleida, Av. Rovira Roure, 80, 25198 Lleida, Spain disease is caused by mutations in a gene on chromosome 9 , called $F X N$ (for frataxin) or $X 25$ that results in decreased frataxin content or expression. In most patients, frataxin deficiency is caused by the presence of a GAA triplet expansion in the first intron of the $F X N$ gene. This expansion is found in both alleles and compromises frataxin expression [3]. Around $4 \%$ of patients are compound heterozygotes for a GAA expansion and a $F X N$ point mutation or deletion [4].

Decreased expression of frataxin is associated with mitochondrial dysfunction, iron and calcium imbalance, and increased oxidative stress. The function of frataxin and the mechanisms causing such cellular disturbances are not completely understood. Frataxin has been shown to localize to the mitochondria, where it regulates the activity of cysteine desulfurase, an enzyme required for the biosynthesis of iron-sulfur clusters [5]. Therefore, it is generally accepted that frataxin activates iron-sulfur biogenesis in eukaryotes. 
Nevertheless, iron-sulfur deficiency is not a universal consequence of frataxin deficiency, suggesting that the role of frataxin in iron-sulfur biogenesis is not essential [6]. Frataxin deficiency also causes mitochondrial iron overload, and different mechanisms have been proposed to explain this fact. First, compromised iron-sulfur biogenesis could interfere with iron sensing by Iron Regulatory Proteins, leading to constitutive activation of iron uptake [7-9]. Second, frataxin binds iron and presents ferroxidase activity, therefore, it could be involved in iron chelation and/or detoxification inside mitochondria [10]. Furthermore, large oligomers of frataxin are formed in vitro in the presence of iron, which could be involved in iron storage. Nevertheless, the in vivo relevance of such structures is controversial (reviewed in [11]). Third, an extramitochondrial form of frataxin has also been described that would bind and regulate Iron Regulatory Protein 1 [12].

Besides iron homeostasis and/or iron-sulfur biogenesis, other processes are altered in frataxin-deficient cells. Several observations suggest that oxidative stress could play a central role in FA. Hypersensitivity to oxidative damage has been observed in FA models and patients, and has been linked to deficient activation of the NRF2 pathway in response to oxidative insults. This pathway is required to induce the expression of antioxidant defenses [13], 14]. The central role of oxidative stress in FA is also highlighted by recent reports indicating that frataxin-deficient cells are hypersensitive to erastin, a drug that causes GSH depletion and is a well-known inducer of ferroptosis $[15,16]$. Ferroptosis is a form of regulated cell death, which is triggered by the accumulation of lipid peroxidation products in membranes [17]. Frataxin deficiency has also been shown to impact different pathways related to mitochondrial function. These include the OXPHOS system [18], 19], mitochondrial calcium efflux [20], mitochondrial permeability pore opening [21], pyruvate dehydrogenase complex [22], and endoplasmic reticulum-mitochondria contacts [23], among others.

Several missense mutations in the frataxin coding region have been identified in FA patients, however, the in vivo consequences of these mutations have hardly been investigated. Galea and collaborators reviewed the consequences of the different frataxin mutations causing FA. Using structural modeling and a systematic literature review they predicted the consequences of these mutations on frataxin structure and function. They also compared clinical information from patients and concluded that there were no significant differences in mean age of onset between GAA-expansion homozygotes and compound heterozygotes carrying missense mutations [24]. This is consistent with observations indicating that the clinical phenotype of patients carrying the I154F mutation is similar to that of individuals homozygous for the GAA expansion [3]. The I154F mutation is one of the most studied frataxin missense mutations. Using cell-free systems and different cellular models, Li and collaborators analyzed the consequences of this mutation on the stability and catalytic activity of mature and intermediate frataxin proteoforms. They observed that I154F compromised the solubility of the intermediate frataxin form and resulted in low levels of mature frataxin. They also observed the presence of insoluble intermediate frataxin proteoforms in cells overexpressing this mutant version of frataxin [25]. From these observations it could be hypothesized that the presence of these insoluble frataxin proteoforms could contribute to the pathology. Besides, I154F mutation has also been reported to inhibit the interaction of frataxin with ISD11 [26] and also to decrease its ability to activate cysteine desulfurase [27].

In the present work we have analyzed the in vivo consequences of the I154F mutation by introducing an I151F substitution into mouse frataxin. This mutation is equivalent to the human I154F pathological mutation. We have observed that $\mathrm{FXN}^{\mathrm{I} 151 \mathrm{~F}}$ homozygous mice present very low frataxin levels, neurological deficits and biochemical alterations which mimic those observed in patients with FA. Therefore, we can conclude that the pathological mechanisms of the I154F mutation are similar to those caused by the GAA triplet expansion and that the $\mathrm{FXN}^{\mathrm{I}}{ }^{151 \mathrm{~F}}$ mouse model is an excellent tool for analyzing the consequences of frataxin deficiency and for testing new therapies.

\section{Materials and methods}

\section{Generation of FXN ${ }^{1151 F}$ mice}

The investigation with experimental animals conforms to the National Guidelines for the regulation of the use of experimental laboratory animals from the Generalitat de Catalunya and the Government of Spain (article 33.a 214/1997) and was evaluated and approved by the Experimental Animal Ethical Committee of the University of Lleida (CEEA). $\mathrm{FXN}^{\mathrm{I} 151 \mathrm{~F}}$ heterozygous mice (C57BL/6 J-Fxn ${ }^{\text {em10(T146T,I151F)Lutzy/J) }}$ were obtained from the Jackson Laboratory, Bar Harbor, ME, USA (Stock Number 31922). To generate these HET mice, the CRISPR + guide targeted mutagenesis reagent was microinjected into C57BL/6 J zygotes. A founder female was crossed with C57BL/6 J to generate N1 HET mice which were also placed into mating to generate N2 HET mice. The incorporation of the $1151 \mathrm{~F}$ point mutation was verified by sequencing the Fxn gene. The mutant allele presented an ATC $\rightarrow$ TTC mutation in codon I151, which causes the expected I151F mutation. An additional silent mutation was present in codon T146 (ACC $\rightarrow$ ACT). The silent T146T mutation was co-introduced with the $1151 \mathrm{~F}$ mutation as a PAM blocker. Intercrosses of HET animals were performed 
to generate the WT, HET and $\mathrm{FXN}^{\mathrm{I} 151 \mathrm{~F}}$ animals used in the present study. Genotyping of these mice was performed by sequencing a PCR product amplified from DNA extracted from tail biopsy specimens. The primers used were Fw: TTT CACACTTCCTGCCACCT; Rv: AGGCAGACAGCCGTA AAGTC. Animals were housed in standard ventilated cages at $12 \mathrm{~h}$ light/dark cycles and fed with a normal chow diet ad libitum. Animals were weighed weekly. For tissue isolation, animals were sacrificed by cervical dislocation at 21 or 39 weeks of age and dissected immediately. Isolated organs were snap-frozen in liquid nitrogen and stored at $-80^{\circ} \mathrm{C}$.

\section{Behavior analyses}

The tests performed were Rotarod, Open Field, Hanging Wire, and Paw Print. All tests were conducted by the same investigator and under dark light conditions. The number of mice used in each analysis is indicated in Supplemental Table 1. Mice were subjected to Rotarod every two weeks from 15 to 39 weeks of age. Open Field, Hanging Wire, and Paw Print tests were performed at 21, 27, 33, and 39 weeks of age. The Rotarod test was performed in a LE 8200 Rotarod system (Panlab Harvard Apparatus).
Animals were subjected to three trials in which, after $1 \mathrm{~min}$ at constant velocity $(5 \mathrm{rpm})$, the rotarod was accelerated to $0.05 \mathrm{rpm} / \mathrm{s}$. The latency to fall after acceleration was recorded and the average of the three trials was the value used. For the Open Field test, a mouse was placed in the center of the field and its movements were recorded during 5 min using Smart Video Tracking software (v2.5.21, Panlab Harvard Apparatus). The parameters measured were total distance travelled, entries in zones (crossings), and average speed. The Hanging Wire test was performed to assess forelimb grip strength and consisted in testing the ability of the mouse to hang on a horizontally positioned wire for $30 \mathrm{~s}$. When the mouse fell from the wire, the latency to fall was recorded. Each mouse had three opportunities to achieve the $30 \mathrm{~s}$ objective. The best score of the three trials was the value used. Bedding material was placed underneath the wire to break the fall. For the Paw Print test, $55 \mathrm{~cm}$ long and $10 \mathrm{~cm}$ wide runway covered with laboratory paper was used. First, each mouse was placed on the runway during $30 \mathrm{~s}$ for habituation. Then, hind and fore paws were painted with pink and blue nontoxic paint, respectively, and the mouse was placed at the beginning of the runway and allowed to walk. The papers

Table 1 Proteins analyzed in the targeted proteomics analysis.

\begin{tabular}{|c|c|c|c|}
\hline Protein Name & Abbrev & Uniprot & Comments \\
\hline Citrate Synthase & $\mathrm{CS}$ & Q9CZU6 & Component of the TCA cycle \\
\hline Mitochondrial Aconitase & $\mathrm{ACO} 2$ & Q99KI0 & Component of the TCA cycle \\
\hline Succinate Dehydrogenase flavoprotein subunit & SDHA & Q8K2B3 & Component of OXPHOS complex II and the TCA cycle \\
\hline Succinate Dehydrogenase iron-sulfur subunit & SDHB & Q9CQA3 & Component of OXPHOS complex II and the TCA cycle \\
\hline Cytochrome c & $\mathrm{CYC}$ & P62897 & Electron carrier involved in OXPHOS \\
\hline Cytochrome $\mathrm{c} 1$, heme protein & CY1 & Q9D0M3 & Component of OXPHOS complex III \\
\hline Cytochrome b-c1 complex subunit 2 & QCR2 & Q9DB77 & Component of OXPHOS complex III \\
\hline Cytochrome c oxidase subunit 2 & $\mathrm{COX} 2$ & P00405 & Component of OXPHOS complex IV \\
\hline ATP synthase subunit alpha & ATPA & Q03265 & Component of OXPHOS complex V \\
\hline ATP synthase subunit beta & ATPB & P56480 & Component of OXPHOS complex V \\
\hline PDH E1 component subunit alpha & PDHA1 & P35486 & Component of the E1 PDH complex \\
\hline Dihydrolipoyllysine-residue acetyltransferase & DLAT & Q8BMF4 & E2 component of the PDH complex \\
\hline Dihydrolipoyl dehydrogenase & DLDH & O08749 & E3 component of the PDH complex \\
\hline Stress-70 protein & GRP75 & P38647 & Mitochondrial Chaperone \\
\hline $60 \mathrm{kDa}$ heat-shock protein & HSP60 & P63038 & Mitochondrial Chaperone \\
\hline Superoxide dismutase $[\mathrm{Cu}-\mathrm{Zn}]$ & SOD1 & P08228 & Cytosolic superoxide dismutase \\
\hline Superoxide dismutase [Mn] & SOD2 & P09671 & Mitochondrial superoxide dismutase \\
\hline Alpha-enolase & ENOA & P17182 & Glycolytic enzyme \\
\hline Beta-enolase & ENOB & $\mathrm{P} 21550$ & Glycolytic enzyme \\
\hline Gamma-enolase & ENOG & P17183 & Glycolytic enzyme \\
\hline Pyruvate kinase PKM & PKM & P52480 & Glycolytic enzyme \\
\hline Glyceraldehyde-3-phosphate dehydrogenase & GAPDH & P16858 & Glycolytic enzyme \\
\hline
\end{tabular}

The abbreviations used throughout this manuscript and the Uniprot accession codes are indicated. Transitions used for detection of each protein are indicated in supplemental information. TCA: Tricarboxylic acid cycle

PDH: Pyruvate dehydrogenase 
were scanned and the stride length of the animals was measured using ImageJ software.

\section{Preparation of tissue homogenates}

A tissue sample (20-100 $\mathrm{mg}$ ) was cut into $2-3 \mathrm{~mm}^{2}$ pieces. All pieces were placed in $1.5 \mathrm{ml}$ screw cap polypropylene tubes in the presence of lysis buffer consisting of $50 \mathrm{mM}$ tris(hydroxymethyl)aminomethane (Tris) $\mathrm{HCl} \mathrm{pH} 7.5$ containing a protease inhibitor cocktail (Roche). For each $100 \mathrm{mg}$ of tissue, $375 \mu \mathrm{l}$ of lysis buffer were used. Glass beads $(0.5-1.0 \mathrm{~mm})$ were added to the mixture, which was then homogenized in a BioSpec Mini-Beadbeater. Following homogenization, SDS was added to a $4 \%$ final concentration. This homogenate was vortexed for $1 \mathrm{~min}$, heated at $98^{\circ} \mathrm{C}$ for $5 \mathrm{~min}$, sonicated and subsequently centrifuged at $12,000 \mathrm{~g}$ for $10 \mathrm{~min}$. Protein content in the supernatant was quantified using the BCA assay (Thermo Scientific). For fractionation of tissue homogenates, tissues were homogenized in the presence of a homogenization buffer consisting of $25 \mathrm{mM}$ HEPES-KOH, pH 7.4, $100 \mathrm{mM} \mathrm{NaCl}$, and protease inhibitor cocktail (Roche). These homogenates were subsequently centrifuged at $20,000 \mathrm{~g}$ for $20 \mathrm{~min}$ at $4{ }^{\circ} \mathrm{C}$, yielding a soluble supernatant and an insoluble sediment. The supernatant was transferred to a new tube and SDS was added to reach a 4\% final concentration. The sediment was washed three times with homogenization buffer and resuspended to the original volume using homogenization buffer containing 4\% SDS. Both fractions were heated at $100{ }^{\circ} \mathrm{C}$ for $3 \mathrm{~min}$ and loaded on SDS-PAGE gels.

\section{Protein quantitation by targeted proteomics}

Tissue homogenates (50 $\mu \mathrm{g}$ of protein) were precipitated with cold acetone (9 volumes) and resuspended in 1\% sodium deoxycholate, $50 \mathrm{mM}$ ammonium bicarbonate. Then, proteins were subjected to reduction by $12 \mathrm{mM}$ DTT and alkylation by $40 \mathrm{mM}$ IAM. Mass spectrometry grade trypsin (SOLu-Trypsin, Sigma) was added to a final enzyme:substrate ratio of 1:50. After overnight digestion at $37^{\circ} \mathrm{C}$, formic acid was added to precipitate sodium deoxycholate. The resulting peptide mix was purified and enriched using $100 \mu$ l Pierce C18 ZipTips. Eluted fraction from the $\mathrm{C} 18 \mathrm{ZipTip}$ was evaporated using a Concentrator Plus (Eppendorf) and peptides were resuspended in 3\% acetonitrile plus $0,1 \%$ formic acid containing a heavy peptide standards mixture. Heavy peptides were obtained from JPT (SpikeTidesTM_L). All peptide samples were analyzed on a triple quadrupole spectrometer (Agilent 6420) equipped with an electrospray ion source. Chromatographic separations of peptides were performed on an Agilent 1200 LC system using a Supelco Bioshell A160 Peptide C18 column $(1 \mathrm{~mm} \times 15 \mathrm{~cm})$. Peptides (up to $15 \mu \mathrm{g}$ of protein digest) were separated with a linear gradient of acetonitrile/water, containing $0.1 \%$ formic acid, at a flow rate of $75 \mu \mathrm{l} / \mathrm{min}$. A gradient from 3 to $60 \%$ acetonitrile in $45 \mathrm{~min}$ was used. The mass spectrometer was operated in multiple reaction monitoring mode. Transitions were obtained from peptide atlas, SRM atlas or Prosit [28] and imported into Skyline software [29], which was also used to analyze results. Once validated and optimized, the SRM assays were used to quantify all the peptides analyzed using scheduled SRM mode in a single run (retention time window, $120 \mathrm{~s}$; cycle time, $1 \mathrm{~s}$ ). Two injections (replicas) were performed per sample, and the light to heavy abundance ratio (raw $\mathrm{L} / \mathrm{H}$ ratio) calculated for each peptide. A very high correlation in the raw $\mathrm{L} / \mathrm{H}$ ratio between both replicas was observed $(\mathrm{R}=0.99$, Supplemental fig. 5). For calculating protein abundance, first the raw $\mathrm{L} / \mathrm{H}$ ratio of each peptide in each replica was divided by the mean raw $\mathrm{L} / \mathrm{H}$ ratio of all the peptides in the same replica. This was performed in order to correct for potential protein loss during sample preparation. Then, this value was divided by the average value of each peptide among all samples and replicas. This value was termed relative $\mathrm{L} / \mathrm{H}$. The final protein abundance value in a sample was obtained by calculating the average value among the relative $\mathrm{L} / \mathrm{H}$ values from the different replicas and peptides corresponding to the same protein and sample. For most proteins analyzed, reproducibility between experimental replicas (different animals from the same experimental condition) was very high, as indicated by coefficients of variance below 10\% (Supplemental Fig. 5B). Peptides and transitions analyzed are shown in. Supplemental Table 2.

\section{Western blot}

After SDS-polyacrylamide gel electrophoresis, proteins were transferred to PVDF (Millipore, IPVH00010) or Nitrocellulose (Sigma_Aldrich, 10,600,093) membranes and blocked with I-block (ThermoFisher, T2015). The membranes were probed with the following primary antibodies: Frataxin (1:1000 Abcam, ab219414; 1:500 Abcam, ab175402; 1:500 Abcam, ab113691; 1:500 MyBioSource, mbs8245785), aconitase 2 (1:15,000 Sigma, HPA001097), lipoic acid (1:20,000 Calbiochem, 437,695) and OxPhos (1:20,000 Invitrogen, 458,099), $\alpha$-Tubulin $(1: 50,000$ Sigma, T5168). The detection was performed using peroxidase conjugated secondary antibodies. Image acquisition was performed in a ChemiDoc MP system from Bio-Rad. Membranes were stained with Coomassie brilliant blue or Ponceau for normalization, where each entire lane densitometry was used. When required, data was analyzed by ImageLab software (Bio-Rad). Apparent MW was calculated using Precision Plus Protein Standards (Bio-Rad) and ImageLab software using the point-to-point semi-log interpolation method. 


\section{Enzyme activities}

A tissue sample was cut into $2-3 \mathrm{~mm}^{2}$ pieces. All pieces were placed into tubes containing nondenaturing lysis buffer consisting of Tris- $\mathrm{HCl} 50 \mathrm{mM} \mathrm{pH} \mathrm{7.4,} \mathrm{protease} \mathrm{inhibitor}$ cocktail (Roche), and sodium citrate $2.5 \mathrm{mM}$. Glass beads $(0.5-1.0 \mathrm{~mm})$ were added and tissues were homogenized using a BioSpec Mini-Beadbeater. Then, Triton X-100 was added to a final concentration of $0.5 \%$. Homogenized tissues were centrifuged at $13,000 \mathrm{rpm}$ at $4^{\circ} \mathrm{C}$ during $5 \mathrm{~min}$ and the supernatants were placed into new tubes. Aconitase activity was measured in Tris- $\mathrm{HCl} 50 \mathrm{mM} \mathrm{pH} 7.4$, containing $1 \mathrm{mM}$ sodium citrate, $0.2 \mathrm{mM}$ NADP, $0.6 \mathrm{mM}$ manganese chloride, and 0.25 units of isocitrate dehydrogenase (Sigma-Aldrich, I2002). NADPH formation was measured at $340 \mathrm{~nm}$ during $120 \mathrm{~s}$. Citrate synthase activity was measured with a coupled assay to reduce 5,5'-dithiobis-(2-nitrobenzoic acid) (DTNB). Briefly, tissue extracts were added to Tris- $\mathrm{HCl} 100 \mathrm{mM}$ pH 8.1 with $0.4 \mathrm{mg} / \mathrm{ml}$ DTNB and $10 \mathrm{mg} / \mathrm{ml}$ Acetyl-CoA. Absorbance was measured at $412 \mathrm{~nm}$ during $120 \mathrm{~s}$. Then, $8.5 \mathrm{mg} / \mathrm{ml}$ of oxaloacetate were added to the cuvette and the absorbance was measured again at $412 \mathrm{~nm}$ during $120 \mathrm{~s}$ for the detection of reduced DTNB. Values are presented as a ratio of aconitase activity versus citrate synthase activity.

\section{Quantitative real-time PCR}

For qRT-PCR analysis, 50-100 $\mu \mathrm{g}$ of tissue sample was homogenized with $1 \mathrm{~mL}$ of TRIzol ${ }^{\mathrm{TM}}$ Reagent (Invitrogen, $15,596,018)$ using an IKA® T10 basic homogenizer. RNA was extracted following manufacturer's instructions. For each sample, $1 \mu \mathrm{g}$ of total RNA was converted into cDNA with the iScript cDNA Synthesis Kit (Bio-Rad, 1,708,891) and $50 \mathrm{ng}$ of cDNA were used in each reaction. The assays were performed in a CFX96 Real-Time System (Bio-Rad) using TaqMan ${ }^{\circledR} 2 \mathrm{X}$ Universal PCR Master Mix (Applied Biosystems, 4,304,437). Real-Time PCR was performed using TaqMan probes: Sdha (Mm01352366_m1), Sdhb (Mm00458272_m1), Ndufb8 (Mm00482663_m1), Aco2 (Mm00475673) and Gapdh (Mm99999915_g1). Gapdh was used as an internal control. Quantification was completed using Bio-Rad CFX Manager real-time detection system software (version 3.1, Bio-Rad). Relative expression ratios were calculated on the basis of $\Delta \mathrm{Cp}$ values with efficiency correction based on multiple samples.

\section{Frataxin overexpression}

HEK $293 \mathrm{~T}$ cells were plated in $35 \mathrm{~mm}$ collagen coated plates at a density of $3 \cdot 10^{5}$ cells/plate. After $20 \mathrm{~h}$, cells were washed once with optiMEM ${ }^{\mathrm{TM}}$ medium (Gibco, 31,985-047) and transfected with a pCMV6-Entry vector containing untagged mouse Fxn ORF (OriGene, NM_008044) using
$10 \mu \mathrm{M}$ of Polyethylenimine in optiMEM ${ }^{\mathrm{TM}}$ medium. Cells were incubated for $1 \mathrm{~h}$ at $37^{\circ} \mathrm{C}$ and then the plasmid was removed and cells were cultured with DMEM (Gibco, 41,966-029) supplemented with $10 \%$ Fetal Bovine Serum. After $24 \mathrm{~h}$, cells were washed once with cold PBS and lysed in $200 \mu \mathrm{l}$ lysis buffer consisting of $125 \mathrm{mM}$ TRIS-HCl pH $7.5,2 \%$ SDS and protease inhibitor cocktail (Roche). Lysates were heated at $100{ }^{\circ} \mathrm{C}$ for $3 \mathrm{~min}$ and loaded on SDS-PAGE gels.

\section{Statistical analysis}

Statistical analyses were performed using GraphPad Prism (version 8). The two-tailed Student's t test was used to assess the significance of the differences between protein and mRNA content data. In mouse analyses, differences between groups were assessed by two-way ANOVA with Benjamini, Krieger and Yekutieli post hoc test. The $\mathrm{p}$ values lower than $0.05(*), 0.01(* *)$ or $0.001(* * *)$ were considered significant.

\section{Results}

\section{Generation of frataxin I151F knock-in mice}

$\mathrm{FXN}^{\mathrm{I} 151 \mathrm{~F} / \mathrm{wt}}$ heterozygous mice (C57BL/6J$\mathrm{Fxn}^{\mathrm{em} 10(\mathrm{~T} 146 \mathrm{~T}, \mathrm{I1} 151 \mathrm{~F}) \mathrm{Lutzy} / \mathrm{J}}$ ) were obtained from the Jackson Laboratory (Stock Number 31922). To generate these mice, CRISPR/Cas9 was used to introduce the ATC $\rightarrow$ TTC mutation into codon I151 of murine Fxn. The resulting I151F substitution is equivalent to the human pathogenic I154F missense mutation (Figure 1A). An additional silent mutation is present in codon T146 (ACC $\rightarrow \mathrm{ACT}$ ). This change was deliberately co-introduced with the desired mutation in order to destroy the guide dependent protospacer adjacent motif (PAM) recognition site. Inter-crossing of these mice resulted in progeny of all possible genotypes. Of 180 mice born, $48(26,7 \%)$ were WT mice (FXN ${ }^{\mathrm{wt} / \mathrm{wt}}$ homozygous for wild type FXN), $91(50,5 \%)$ were HET mice $\left(\mathrm{FXN}^{\mathrm{I151F} / \mathrm{wt}}\right.$, heterozygous for wild type FXN and FXNI151F), and 41 $(22,8 \%)$ were $\mathrm{FXN}^{\mathrm{I} 151 \mathrm{~F}}$ mice $\left(\mathrm{FXN}^{\mathrm{I} 151 \mathrm{~F} / 151 \mathrm{~F}}\right.$, homozygous for the FXNI151F allele) (Figure 1D). Therefore, progeny was within the expected mendelian ratios indicating that $\mathrm{FXN}^{\mathrm{I151F}}$ mice were viable.

\section{Frataxin protein levels are markedly reduced in $\mathrm{FXN}^{1151 \mathrm{~F}}$ mice}

To determine the effect of the $1151 \mathrm{~F}$ mutation on frataxin content, we measured the levels of this protein in cerebrum, cerebellum and heart from 21 and 39-week-old WT, HET and FXN ${ }^{\mathrm{I151F}}$ mice. By western blot (using monoclonal antibody Ab219414), we observed that the 


\section{A}

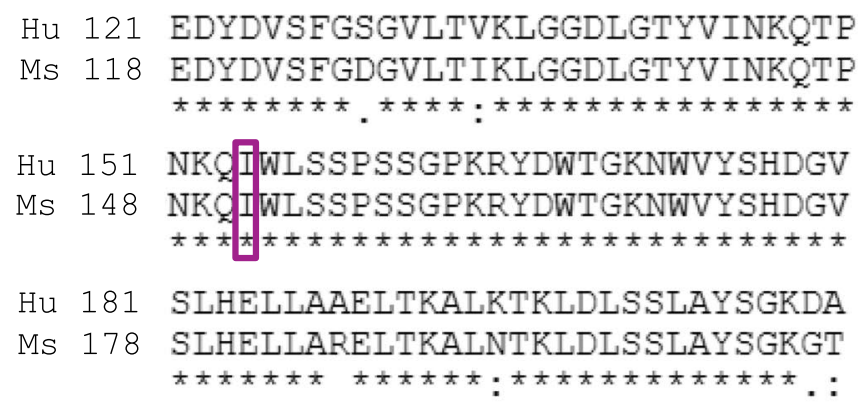

B

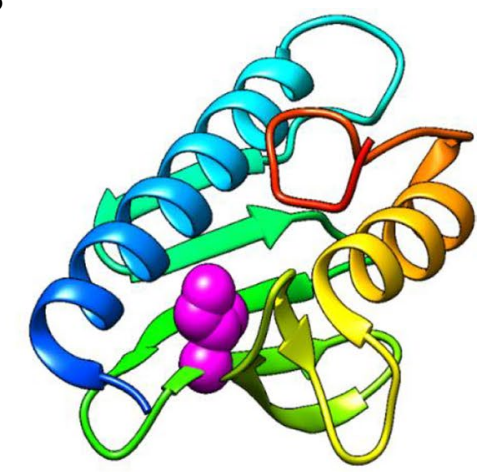

\section{C}

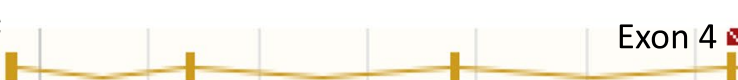

Exon 4 :
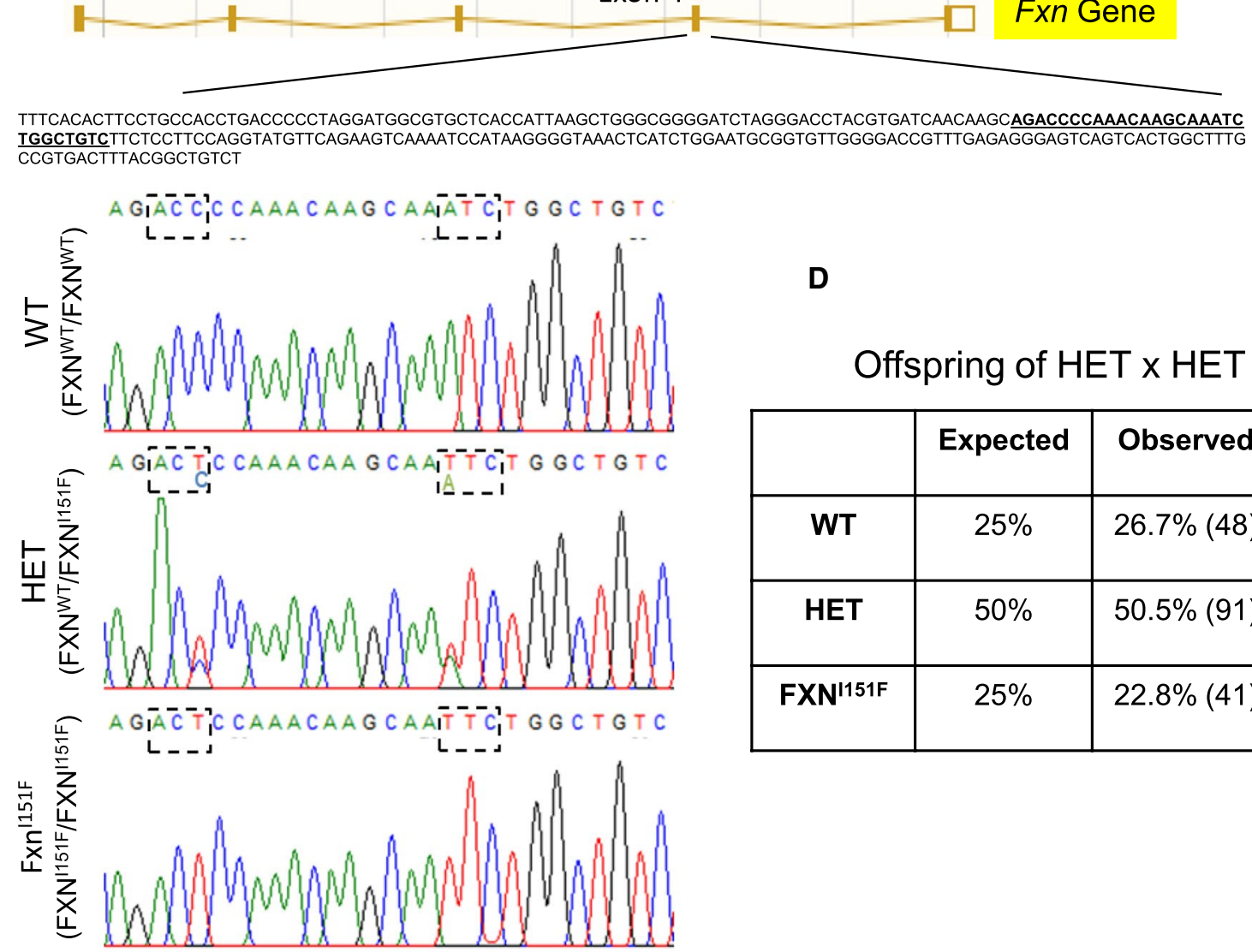

D

Offspring of HET $\times$ HET

\begin{tabular}{|c|c|c|}
\hline & Expected & Observed \\
\hline WT & $25 \%$ & $26.7 \%(48)$ \\
\hline HET & $50 \%$ & $50.5 \%(91)$ \\
\hline FXN $^{1151 F}$ & $25 \%$ & $22.8 \%(41)$ \\
\hline
\end{tabular}

Fig. 1 Generation of the $\mathrm{FXN}^{\mathrm{I} 151 \mathrm{~F}}$ model. A Alignment of the C-terminal region from human (Hu, Uniprot Q16595) and mouse (Ms, Uniprot O35943) frataxin protein sequences. Amino acid I151 in mice corresponds to I154 in humans (boxed). B ribbon representation of human frataxin (pdb code $3 \mathrm{~s} 4 \mathrm{~m}$ ) showing the position of I154 (spacefill, in violet). Ribbons are colored according to sequence, from dark blue ( $\mathrm{N}$-terminal) to red (C-terminal). Molecular graphics were performed with the UCSF Chimera package. C DNA sequence

content of mature frataxin in HET mice was approximately $50 \%$ of that observed in WT mice, while less than $6 \%$ of mature frataxin content was observed in $\mathrm{FXN}^{\mathrm{I} 151 \mathrm{~F}}$ mice analyses of WT, HET, and FXN ${ }^{\mathrm{I151F}}$ mice. The top panel shows a schematic diagram of the Fxn Gene. The amplified sequence used in genotyping assays includes exon 4 and is indicated. The region containing the mutations is highlighted and shown below. Dashed boxes indicate the $\mathrm{ACC} \rightarrow \mathrm{ACT}$ (silent) and $\mathrm{ATC} \rightarrow \mathrm{TTC}(\mathrm{I} 151 \mathrm{~F})$ mutations introduced by gene editing into the frataxin gene. D Genotyping of litters from HET intercrosses

(Fig. 2A and supplemental Figure 1B). We also analyzed the frataxin content in homogenates from spinal cord, dorsal root ganglia (DRG), liver, pancreas and skeletal 
A
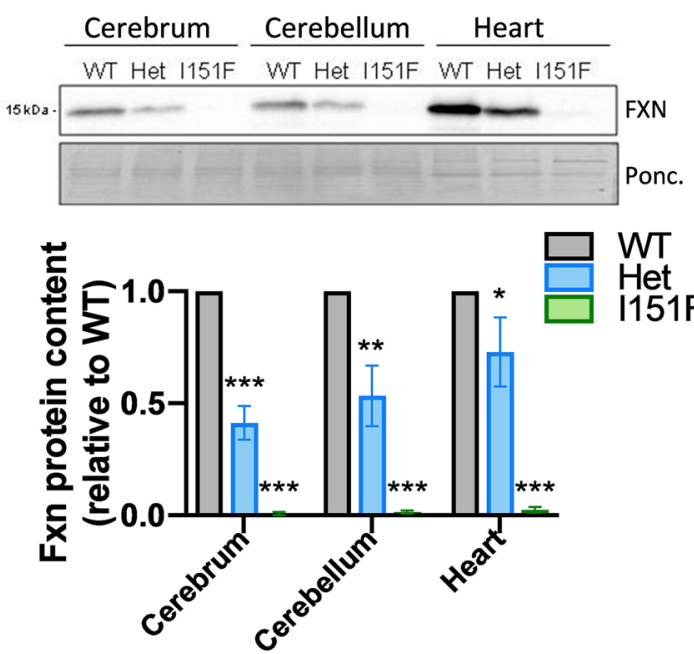

39 weeks
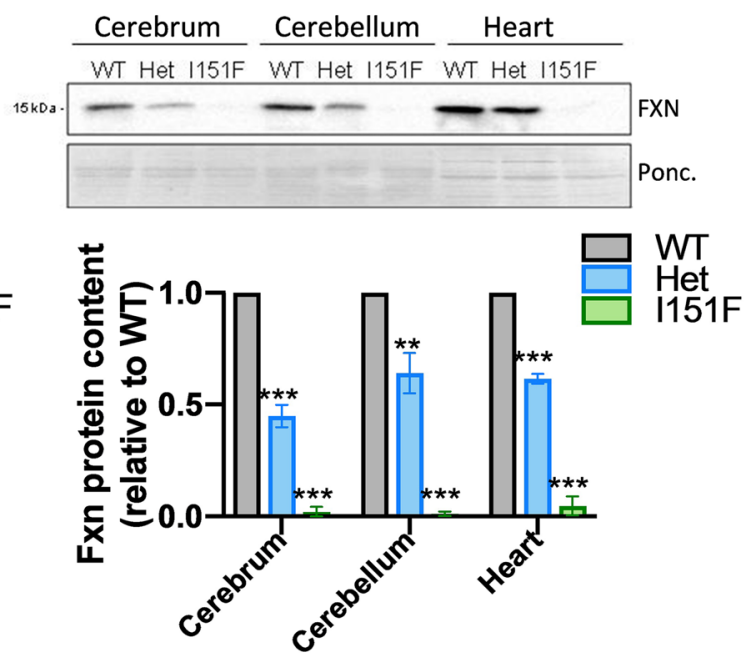

B
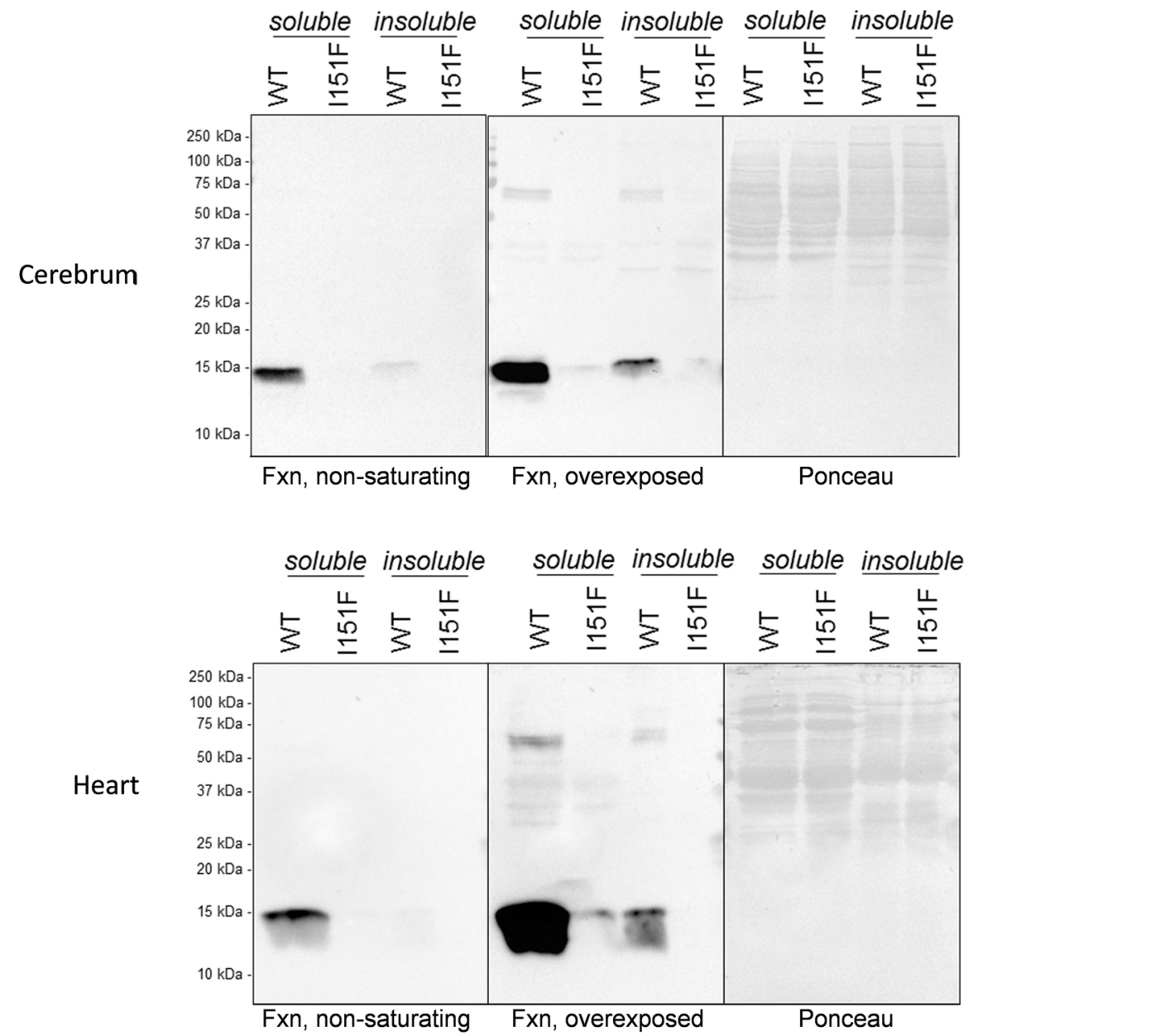

Fig. $2 \mathrm{FXN}^{\mathrm{I1} 151 \mathrm{~F}}$ mice present decreased frataxin content. A Relative mature frataxin $(15 \mathrm{kDa})$ content analyzed by western blot in cerebrum, cerebellum, and heart homogenates from WT, HET, and FXN ${ }^{1151 F}$ mice. B Cerebrum and heart homogenates from 21-weekold WT and FXN ${ }^{\mathrm{I151F}}$ mice were prepared under native conditions and the soluble and insoluble protein fractions separated by centrifugation. Representative images from frataxin western blot membranes under nonsaturating or overexposed conditions are shown. Whole membrane is shown to indicate the absence of insoluble full-length or intermediate frataxin proteoforms in $\mathrm{FXN}^{\mathrm{I} 151 \mathrm{~F}}$ samples 
muscle. These analyses confirmed that the I151F mutation causes a marked loss of frataxin content in all mouse tissues (Supplemental Figure 1). In order to exclude the possibility that the decreased frataxin signal in the western blot was caused by a loss of epitope recognition by the monoclonal antibody employed, three additional antibodies were used to detect frataxin in cerebrum homogenates. These were two polyclonal antibodies raised against full-length frataxin (Mbs8245785 and Ab175402), and a monoclonal antibody (Ab113691) raised against a peptide within aa 1-120 from human frataxin (thus not including I154). Also, we overexpressed murine frataxin in HEK293 cells in order to identify the migration pattern of mature, intermediate and frataxin proteoforms. These results are shown in supplemental figure $2 \mathrm{~A}$. Three major bands can be overserved in overexpressing cultures frataxin: the slower migrating band has an apparent molecular weight of $26.6 \mathrm{kDa}$, and corresponds to the precursor form (theoretical MW $22.9 \mathrm{kDa}$ ); the intermediate form has an apparent MW of $18.1 \mathrm{kDa}$ (theoretical MW 18.6 kDa); the mature form has an apparent MW of $15.3 \mathrm{kDa}$ (theoretical MW 14.4 kDa). Regarding frataxin detection in cerebrum homogenates with the different antibodies used, all of them were able to detect a band migrating at $15 \mathrm{kDa}$ in WT samples. This band disappeared in I151F samples, confirming that the mature frataxin content in $\mathrm{FXN}^{\mathrm{I} 151 \mathrm{~F}}$ mice is very low. The Mbs8245785 and Ab113691 antibodies recognized bands in the 20-26 kDa range, but these bands may be caused by cross-reaction of the antibody with unknown proteins, as they were not detected by antibodies Ab219414 and Ab175402. To further confirm the frataxin decrease in $\mathrm{FXN}^{\mathrm{I} 151 \mathrm{~F}}$ mice, we used a mass spectrometry-based targeted proteomics SRM approach to detect frataxin in tissues collected from WT and FXN ${ }^{\mathrm{I1} 51 \mathrm{~F}}$ mice. This approach was based on the detection of proteotypic peptides from frataxin in trypsin-digested tissue homogenates, which were analyzed in a liquid chromatograph coupled to a triple quadrupole mass spectrometer (proteotypic peptides are those peptides in a protein that are most likely to be observed by mass spectrometry and which are unique to that protein). We selected the four peptides with higher ESS score in peptideatlas server (ESS provides an estimation of the proteotypicity of each peptide) and for each one of these we selected the three most frequently observed transitions. These transitions are indicated in Supplemental Figure 2B. All these peptides are present in both mature, precursor and intermediate forms of frataxin. Using this approach, we were able to detect a signal in heart homogenates eluting at minute 22.7, corresponding to the $2+$ to $y 7+$ transition of the LGGDLGTYVINK peptide. We could not detect this signal in cerebrum or cerebellum homogenates, probably because frataxin is less abundant in the nervous system than in heart (as observed in figure 2A). None of the other peptides analyzed were detected. It is worth mentioning that frataxin is not easily detected by mass spectrometry as according to peptide atlas frataxin has been observed 386 times, while mitochondrial aconitase has been observed 26660 times. In order to confirm that the signal observed in heart homogenates corresponded to the LGGDLGTYVINK peptide, an isotopically labeled heavy version of the peptide was purchased and included in the analysis as an internal standard. This heavy form presented the same retention time as the light form. Moreover. the light form was not detected in heart from FXN ${ }^{\mathrm{I} 151 \mathrm{~F}}$ mice (Supplemental figure $2 \mathrm{C}$ ), further confirming that frataxin was markedly decreased in these animals. Due to its low intensity, we were not able to accurately quantify the residual frataxin present in mutant mice, but we could estimate that it was below $5 \%$ of WT values. This value is consistent with the western blot data and confirms that frataxin is markedly decreased in $\mathrm{FXN}^{\mathrm{I} 151 \mathrm{~F}}$ mice. These results also indicate that intermediate or precursor proteoforms are not detected in neither WT or FXN ${ }^{1151 F}$ mice.

\section{Insoluble intermediate frataxin proteoforms are not detected in FXN ${ }^{1151 F}$ mice}

The presence of an insoluble intermediate frataxin proteoform has been reported in cells overexpressing human I154F frataxin [25]. Results presented in the previous section suggest that this form may not be present in $\mathrm{FXN}^{\mathrm{I} 151 \mathrm{~F}}$ mice, as the denaturing conditions used for protein extraction in these assays should solubilize such intermediate insoluble forms. Nevertheless, in order to confirm this point, we analyzed the potential presence of this insoluble form under the same conditions used to detect human insoluble FXNI154F. To this purpose, we prepared tissue homogenates under native conditions, and we separated the soluble protein fraction from the insoluble protein fraction by centrifugation. After solubilizing the insoluble fraction in a buffer containing $4 \%$ SDS (a concentration high enough to solubilize the previously reported I154F insoluble intermediate frataxin form) both fractions were loaded on SDS-PAGE gels and frataxin proteoforms detected by western blot (antibody Ab219414). Membranes were overexposed in order to detect any potential intermediate or high molecular weight forms of frataxin. In these membranes, we could detect the presence of some high molecular weight bands in WT homogenates (Figure 2B). Nevertheless, these high molecular weight forms represented less than $1 \%$ of the chemiluminescent signal from the mature form, and were also decreased in homogenates from $\mathrm{FXN}^{\mathrm{I} 151 \mathrm{~F}}$ mice. Therefore, we can exclude the presence of an insoluble intermediate frataxin proteoform in mice harboring the I151F mutation. 


\section{$\mathrm{FXN}^{1151 F}$ mice present decreased weight gain}

Body weight of $\mathrm{WT}$ and $\mathrm{FXN}^{\mathrm{I} 151 \mathrm{~F}}$ mice was measured every two weeks from birth. Weight gain was similar in WT and FXN ${ }^{\mathrm{I} 151 \mathrm{~F}}$ mice until 10 weeks of age. From that age on, weight gain was lower in $\mathrm{FXN}^{\mathrm{I} 151 \mathrm{~F}}$ mice than in WT mice. Therefore, significant weight differences were observed between WT and FXN ${ }^{\mathrm{I} 151 \mathrm{~F}}$ mice from 15 weeks of age onward (Figure 3A and B). The relative weight difference between $\mathrm{WT}$ and $\mathrm{FXN}^{\mathrm{I} 151 \mathrm{~F}}$ mice was progressive and was similar in both females and males. At 39 weeks of age, $\mathrm{FXN}^{\mathrm{I} 151 \mathrm{~F}}$ mice presented on average a $23 \%$ decrease in weight when compared with WT mice (Figure 3C and D). HET mice did not present any weight loss (Supplemental Figure 3A).

\section{FXN $^{1151 F}$ mice exhibit neurological deficits}

To determine whether frataxin deficiency caused by the $\mathrm{I} 151 \mathrm{~F}$ mutation impacted the behavior of $\mathrm{FXN}^{\mathrm{I} 151 \mathrm{~F}}$ mice, we subjected these mice to several motor behavioral tasks. WT mice were also analyzed as a control. The number of mice used for each analysis is summarized in Supplemental Table 1 . The motor coordination ability was assessed on a rotarod treadmill. This analysis was performed every two weeks, from 15 to 39 weeks of age. As shown in figure 4A, $\mathrm{FXN}^{\mathrm{I} 151 \mathrm{~F}}$ mice showed decreased coordination ability compared with WT. Differences were statistically significant
A

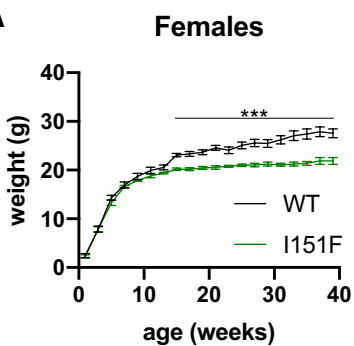

C

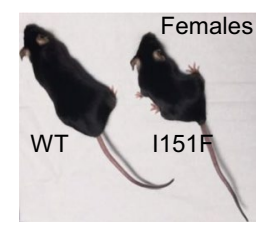

B

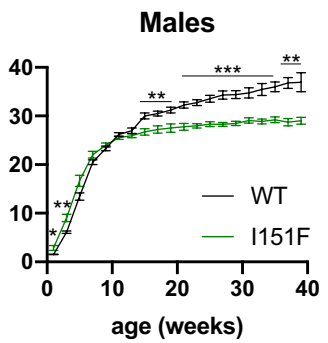

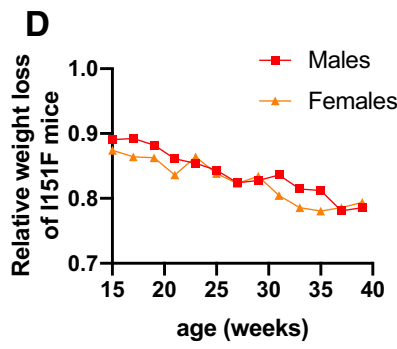

Fig. 3 FXN $^{\mathrm{I} 151 \mathrm{~F}}$ mice present decreased weight gain. A and $\mathbf{B}$ Body weight of WT and $\mathrm{FXN}^{\mathrm{I}}{ }^{51 \mathrm{~F}}$ mice up to 39 weeks of age. C Representative image from WT and $\mathrm{FXN}^{\mathrm{I} 151 \mathrm{~F}}$ female mice. Values are shown as mean \pm SEM. D Average weight loss of FXN ${ }^{1151 \mathrm{~F}}$ mice relative to WT mice of the same age. Number of animals used in each analysis is indicated in supplemental table 1 from week 23 onward. Forelimb strength was tested using a hanging-wire test, where mice were assessed for their ability to hang on a horizontal wire by their forepaws. Analyses were performed every six weeks (starting at week 21). $\mathrm{FXN}^{\mathrm{I151F}}$ mice fell off the wire quicker than WT mice. Statistically significant differences were obtained for 27-, 33-, and 39-week-old mice (Figure 4B). Locomotor activity tests were performed using an open-field beam-breaker activity monitor. These analyses were performed every six weeks (starting at week 21). $\mathrm{FXN}^{\mathrm{I} 151 \mathrm{~F}}$ mice exhibited significantly reduced average velocity, ambulatory distance (total distance covered by the mice within a specific time), and number of crossings than WT mice (Figure 4C). Finally, we assessed gait ataxia using paw print analysis every six weeks (starting week 21). Significant differences between $\mathrm{FXN}^{\mathrm{I} 151 \mathrm{~F}}$ and WT mice were detected in 39-week-old mice, but not before. At this age, $\mathrm{FXN}^{\mathrm{I} 151 \mathrm{~F}}$ mice displayed reduced hind and front limb stride length compared with WT. This suggests that $\mathrm{FXN}^{\mathrm{I} 151 \mathrm{~F}}$ mice present a progressive ataxic gait (Figure 4D). Overall, the results presented in this section indicate that $\mathrm{FXN}^{\mathrm{I} 151 \mathrm{~F}}$ mice present progressive neurologic defects that are not observed before 23 weeks of age. Finally, in order to explore the effect of the I151F mutation in heterozygosis, we analyzed the performance of HET mice in an open field test. No significant differences were observed in the open field test between WT and HET mice, neither in velocity, distance travelled, nor number of crossings (Supplemental Figure 3B).

\section{Analysis of frataxin-related proteins by targeted proteomics}

We were interested in analyzing the biochemical consequences of frataxin deficiency in nervous and cardiac tissues before (21-weeks) and after the onset of neurological defects (39-weeks). With this purpose, we decided to focus on several proteins or pathways that have been related to frataxin. In this regard, frataxin deficiency has been described to cause loss of iron-sulfur containing proteins [30], decreased function of the OXPHOS system [19], changes in the content of superoxide dismutases [31], and decreased content of the pyruvate dehydrogenase component PDH A1 [22]. Also, frataxin has been reported to interact with components of the iron-sulfur biosynthesis machinery [32], the OXPHOS system [33], and with the mitochondrial chaperone GRP75 $[26,34]$. Therefore, in order to explore these pathways in $\mathrm{FXN}^{\mathrm{I} 151 \mathrm{~F}}$ mice, we set up a targeted proteomics method to analyze the content of proteins related to these pathways in tissues collected from WT and $\mathrm{FXN}^{\mathrm{I}}{ }^{151 \mathrm{~F}}$ mice. We used an SRM-based targeted proteomics approach in which proteotypic peptides from each protein were detected in a LCtriple quadrupole mass spectrometer. This approach allows the quantitation of several proteins in multiple samples 
Fig. 4 Neurological deficits in FXN ${ }^{\mathrm{I151F}}$ mice. Rotarod, Open field, hanging wire, and Paw print analysis in WT and FXN $^{1151 F}$ animals; A Rotarod test in mice up to 39 weeks of age. $\mathrm{FXN}^{\mathrm{I} 151 \mathrm{~F}}$ animals presented a decreased latency to fall from 21 weeks onward. B Hanging wire test in mice up to 39 weeks of age. FXN ${ }^{1151 \mathrm{~F}}$ animals presented a decreased latency to fall from 27 weeks onward. C Open field test. $\mathrm{FXN}^{\mathrm{I} 151 \mathrm{~F}}$ mice showed significant decline in velocity, total distance traveled, and number of crossings from 33 weeks onward. D Gait footprint analysis revealed abnormalities in walking patterns in $\mathrm{FXN}^{\mathrm{I} 151 \mathrm{~F}}$ mice, which displayed significantly reduced stride length at 39 weeks of age. Representative walking footprint patterns are shown, in which pink and blue marks represent hind and fore paws respectively. Values are shown as mean \pm SEM. Number of animals used in each analysis is indicated in Supplemental Table 1
A

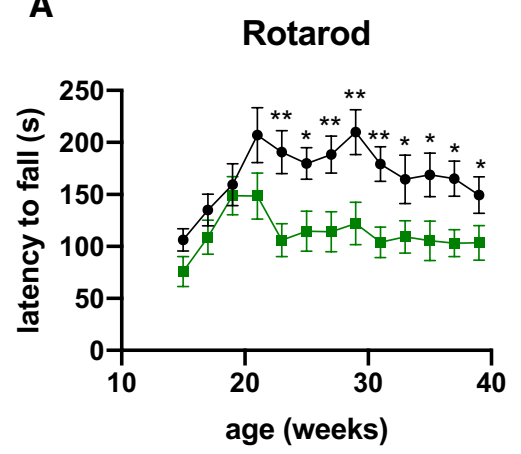

B
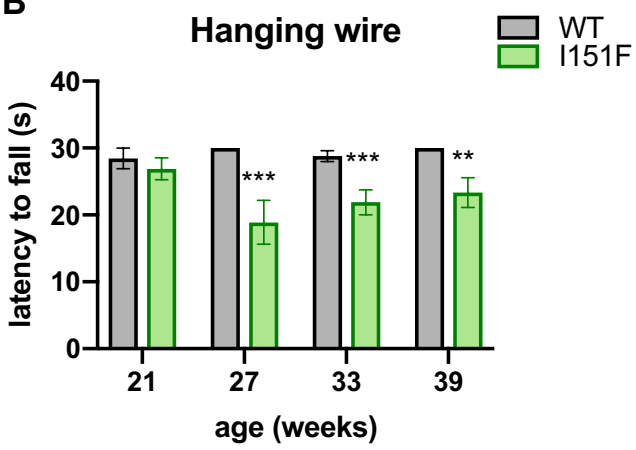

$151 \mathrm{~F}$

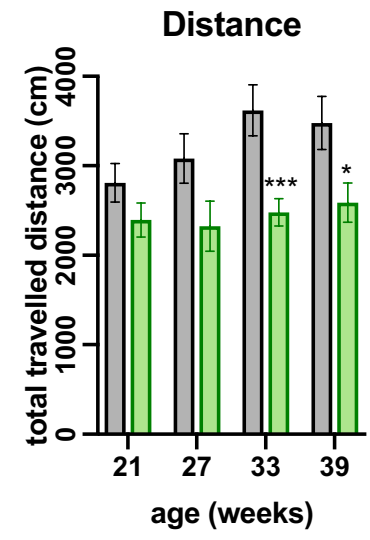

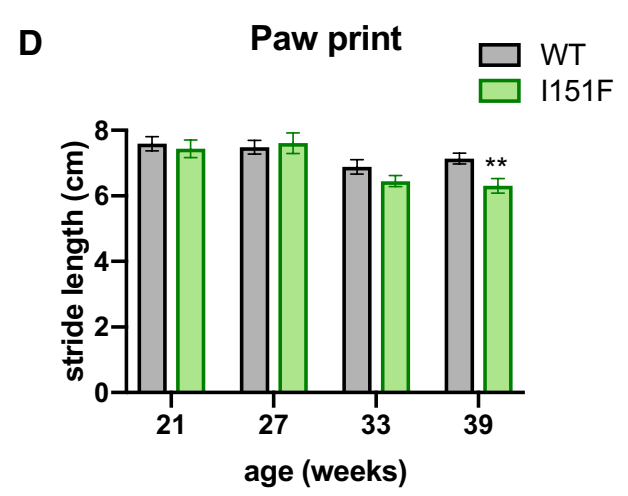

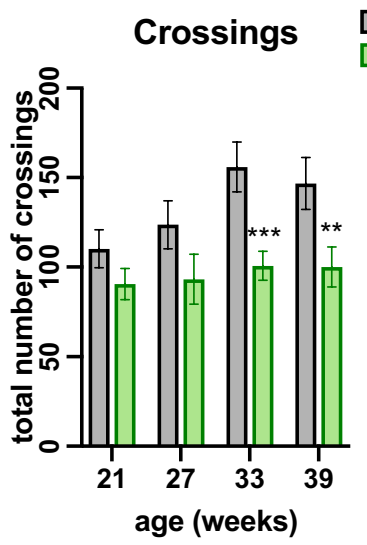

WT I151F
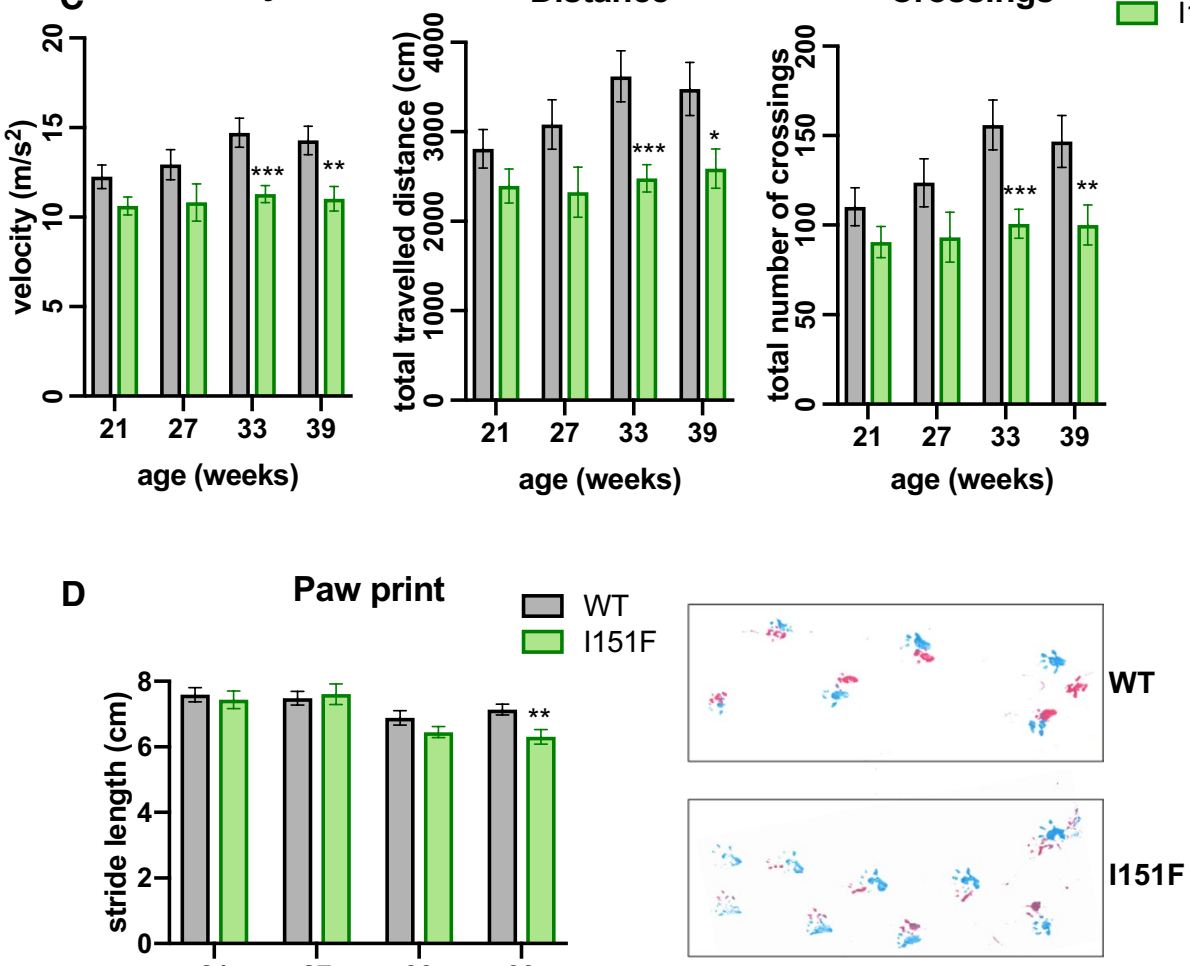

with high reproducibility. The proteins analyzed are listed in Table 1. They consisted of two components of the tricarboxylic acid cycle (CS and ACO2), several components of the OXPHOS system (SDHA, SDHB, CY1, QCR2, COX2, ATPA, and ATPB), two mitochondrial chaperones (HSP60 and GRP75), three components of the Pyruvate dehydrogenase complex (PDHA1, DLAT and DLDH), and two superoxide dismutases (SOD1 and SOD2). Three glycolytic enzymes were also included in the analysis (GAPDH, PKM, and Enolases A, B, G) in order to analyze a potential imbalance between respiratory and glycolytic pathways and/ or between mitochondrial and cytosolic pathways. Two of the proteins analyzed contain iron-sulfur clusters (ACO2 and SDHB), while two of them (CYC and CY1) contain heme groups. The relative content of these 21 proteins was analyzed in cerebrum, cerebellum, and heart from WT and FXN ${ }^{1151 F}$ mice, sacrificed at 21 or 39 weeks of age (in the case of the enolases, ENOG only in nervous systems and ENOB only in heart). Figure 5A-C summarizes the most relevant findings while Supplemental Figure 4 contains the results from all the proteins analyzed. It can be appreciated that the most affected proteins were $\mathrm{ACO} 2$ and the two components of the OXPHOS complex II (SDHA and SDHB). These proteins showed a marked decrease in cerebellum and cerebrum from $\mathrm{FXN}^{\mathrm{I} 151 \mathrm{~F}}$ mice, both at 21 and 39 weeks. In heart, we could only observe loss of complex II in 21-weekold mice, but not in 39-week-old mice, while $\mathrm{ACO} 2$ content was not altered in heart at any age. Some other components 

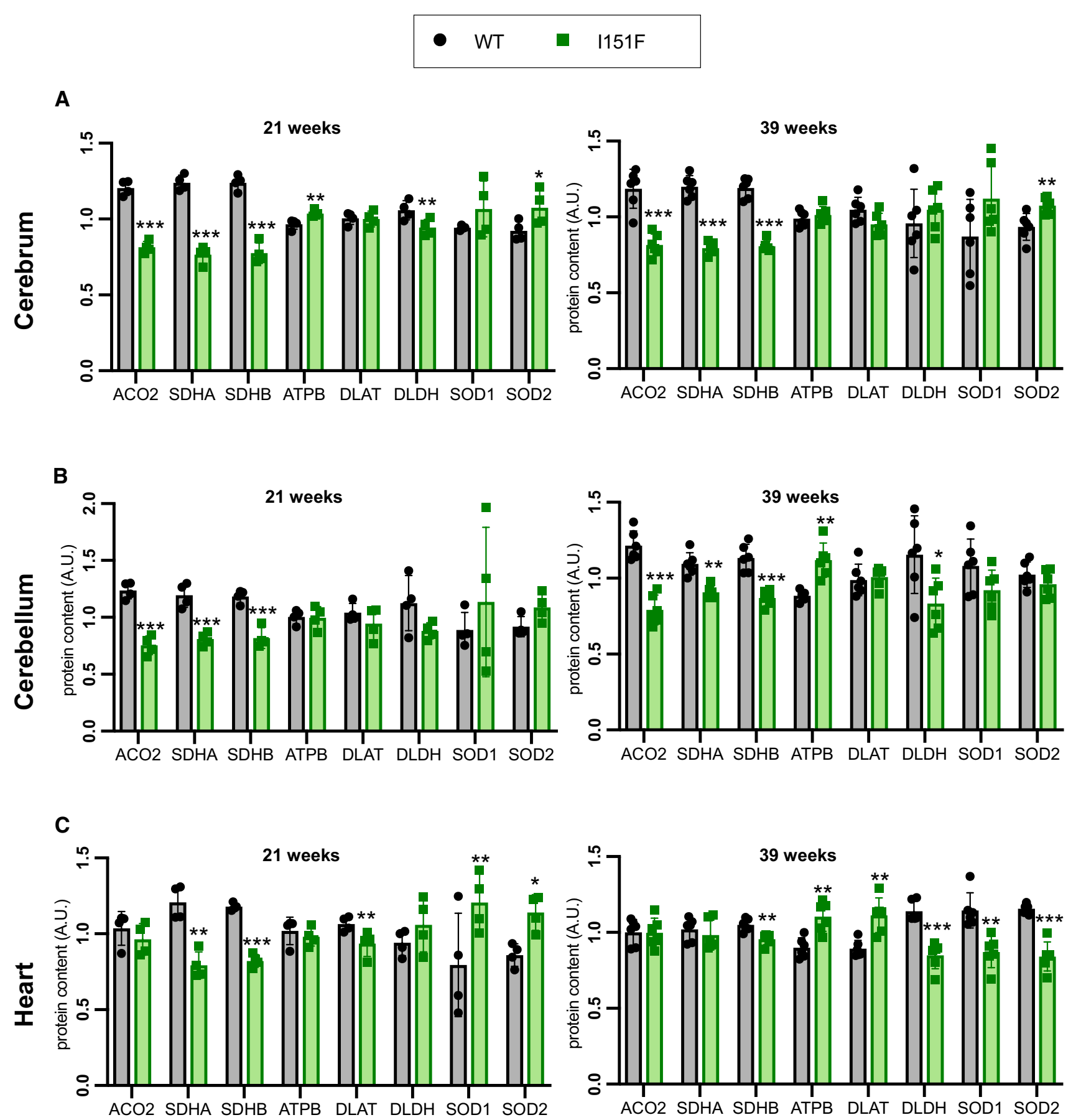

Fig. 5 Analysis of the content of frataxin-related proteins by targeted proteomics. Histograms A to $\mathrm{C}$ show the relative content (in arbitrary units) of the proteins that present the most marked changes by targeted proteomics in either cerebrum, cerebellum, or heart from 21-

of the OXPHOS system presented minor changes: the complex III components QCR2 and CY1 were decreased in cerebrum (21 weeks), cerebellum (21 and 39 weeks), and heart (21 weeks); ATPA and ATB were increased in 39-week-old cerebellum and heart. A second group of proteins showing changes in their content were the antioxidant enzymes SOD1 and 39-week-old WT and FXN ${ }^{1151 F}$ mice. Data are represented as mean \pm SD from four (21-week) or six (39-week) mice. Significant differences $\mathrm{p}$ values $<0.05(*), 0.01(* *)$, or $0.001(* * *)$ between WT and $\mathrm{FXN}^{\mathrm{I} 151 \mathrm{~F}}$ mice are indicated

and SOD2. In heart, both enzymes were induced in 21-weekold mice, while their levels decreased in 39-week-old mice. Some induction of SOD2 was also observed in cerebrum at 21 and 39 weeks. Regarding PDH components, induction of both PDHA1 and DLAT was found in 39-week-old heart. In contrast, DLDH (the E3-component of the PDH 
complex) was decreased in 39-week-old heart and cerebellum. No major changes were observed in the content of the mitochondrial chaperones analyzed, nor of the glycolytic enzymes. The net representation shown in Supplemental Figure 4 allows a comparison of the relative content of the proteins (ratio $\mathrm{FXN}^{\mathrm{I} 151 \mathrm{~F}} / \mathrm{WT}$ ) at 21 and 39 weeks of age. Overall, these results indicate that frataxin deficiency does not cause a general loss of mitochondrial proteins. Instead, it causes specific changes in the content of certain proteins. Also, the net representation indicates how these changes evolve as mice age. In this regard, cerebrum and cerebellum do not experience major differences in the $\mathrm{FXN}^{\mathrm{I} 151 \mathrm{~F}}$ /WT protein ratio between 21 -week-old and 39-week-old mice. Therefore, biochemical alterations precede the neurological defects described in the previous section. In contrast, the heart shows differences in the $\mathrm{FXN}^{\mathrm{I} 151 \mathrm{~F}} / \mathrm{WT}$ protein ratio between 21- and 39-week-old mice, suggesting that the consequences of frataxin loss in this tissue are progressive. Finally, we analyzed the content of some representative proteins in cerebrum, cerebellum, and heart from HET mice. The proteins analyzed were $\mathrm{CS}, \mathrm{ACO} 2$, SDHB, ATPB, SOD2, DLAT, GRP75, and PKM. This group includes representative proteins that experienced changes in the mutant mice (ACO2, SDHB, ATPB and SOD2), and also proteins that did not experience any changes, but are representative of different pathways/functions. These proteins were analyzed in cerebrum, cerebellum, and heart from 39-week-old mice by targeted proteomics. None of these proteins presented relevant changes in their content comparing HET and WT animals (results shown in Supplemental Figure 3). A slight decrease in the amounts of CS and SDHB was observed in the cerebellum of HET mice, but these changes were lower than those observed in $\mathrm{FXN}^{\mathrm{I} 151 \mathrm{~F}}$ mice and/or were not observed in cerebrum and heart.

\section{FXN $^{1151 F}$ mice present lower aconitase activity}

In order to validate the results of the targeted proteomics analysis, we decided to measure the content, activity, and/or expression levels of those proteins most markedly altered in the proteomic analysis. We first focused on $\mathrm{ACO} 2$, a mitochondrial iron-sulfur enzyme, which converts citrate to isocitrate in the tricarboxylic acid cycle. As this enzyme is one of the most abundant iron-sulfur containing proteins, it is commonly used as an indicator of the status of iron-sulfur centers in the cell. Therefore, we analyzed its content by western blot and its activity by spectrophotometry. These analyses were performed in cerebrum, cerebellum, and heart homogenates from 21-week and 39-week-old mice. ACO2 is expected to account for most of the aconitase activity in these tissues as it represents between 80 and $90 \%$ of all aconitase content according to the PaxDb database. Western blot analysis confirmed the results observed in the targeted proteomic analysis, as the results obtained were similar to those obtained in the previous section: a marked decrease was observed in the nervous system, while in heart tissue, no significant differences were observed between WT and $\mathrm{FXN}^{\mathrm{I}}{ }^{151 \mathrm{~F}}$ mice (Figure 6). Regarding activity, we measured the ratio between aconitase and citrate synthase activities (ACO/CS ratio), which was used as a control for non-ironsulfur mitochondrial enzyme. We observed a significantly decreased ACO/CS activity ratio in the three tissues tested, with more marked decreases in cerebrum and cerebellum than in heart (Figure 6). Overall, the results obtained confirm that aconitase content and activity is more affected in the nervous system than in heart. They also suggest that decreased aconitase activity is mostly due to decreased $\mathrm{ACO} 2$ protein content, although the presence of some inactivated protein cannot be excluded (as in some of the conditions analyzed, loss of activity is slightly higher than loss of protein content).

\section{FXN ${ }^{1151 F}$ mice present decreased content of OXPHOS complexes I and II}

The targeted proteomics analysis also revealed changes in the content of several components of the OXPHOS system, notably complex II. In order to confirm these results with an alternative approach, we used western blot to analyze the content of five components of the complexes I to $\mathrm{V}$ (one protein per complex) in cerebrum, cerebellum, and heart from 21- and 39-week-old mice. These proteins were NDUB8 from complex I, SDHB (complex II), QCR2 (complex III), COX1 (complex IV), and ATPA (complex $\mathrm{V})$. None of the complex I components had been included in the targeted proteomics analysis as they could not be properly quantified. The results obtained are shown in Figure 7. It can be observed that $\mathrm{FXN}^{\mathrm{I} 151 \mathrm{~F}}$ mice present a marked loss of NDUB8 from complex I and SDHB from complex II in all tissues analyzed (except heart at 39 weeks), and that this decreased content was already observed at 21 weeks of age. The results from SDHB are similar to those found in the targeted proteomics analysis, confirming the validity of the data obtained. Regarding complexes III to V, a small decrease in QCR2 content (from complex III) could be appreciated in cerebellum, as previously observed in the targeted proteomics approach. No changes in ATPA content were observed by western blot analysis. Overall, the results obtained by the targeted proteomics approach and the western blot analysis indicate that $\mathrm{FXN}^{\mathrm{I}}{ }^{151 \mathrm{~F}}$ mice present a deficiency in complex I and II components of the OXPHOS system, especially in the nervous system, while the components of the other complexes do not present major differences. 
Fig. 6 Aconitase content and activity in $\mathrm{WT}$ and $\mathrm{FXN}^{\mathrm{I} 151 \mathrm{~F}}$ mice. ACO2 content was analyzed by western blot in cerebrum, cerebellum, and heart homogenates from 21 -week (A) and 39-week (D) WT and $\mathrm{FXN}^{\mathrm{I1} 151 \mathrm{~F}}$ mice. Representative western blot images are shown in $\mathbf{A}$ and $\mathbf{D}$. Samples from three different mice were loaded on gels. Histograms $\mathbf{B}$ and $\mathbf{E}$ represent the mean \pm SD from at least four independent measurements; $\mathbf{C}$ and $\mathbf{F}$ Aconitase to Citrate synthase activity ratio (ACO/CS) was measured in cerebrum, cerebellum, and heart homogenates from 21-week $\mathbf{C}$ and 39-week F WT and $\mathrm{FXN}^{\mathrm{I} 151 \mathrm{~F}}$ mice. Data represent the mean \pm SD from at least five different mice. Significant differences between WT and $\mathrm{FXN}^{\mathrm{I} 151 \mathrm{~F}}$ mice are indicated $(\mathrm{p}$ values $<0.05(*), 0.01(* *)$ or $0.001(* * *)$
A
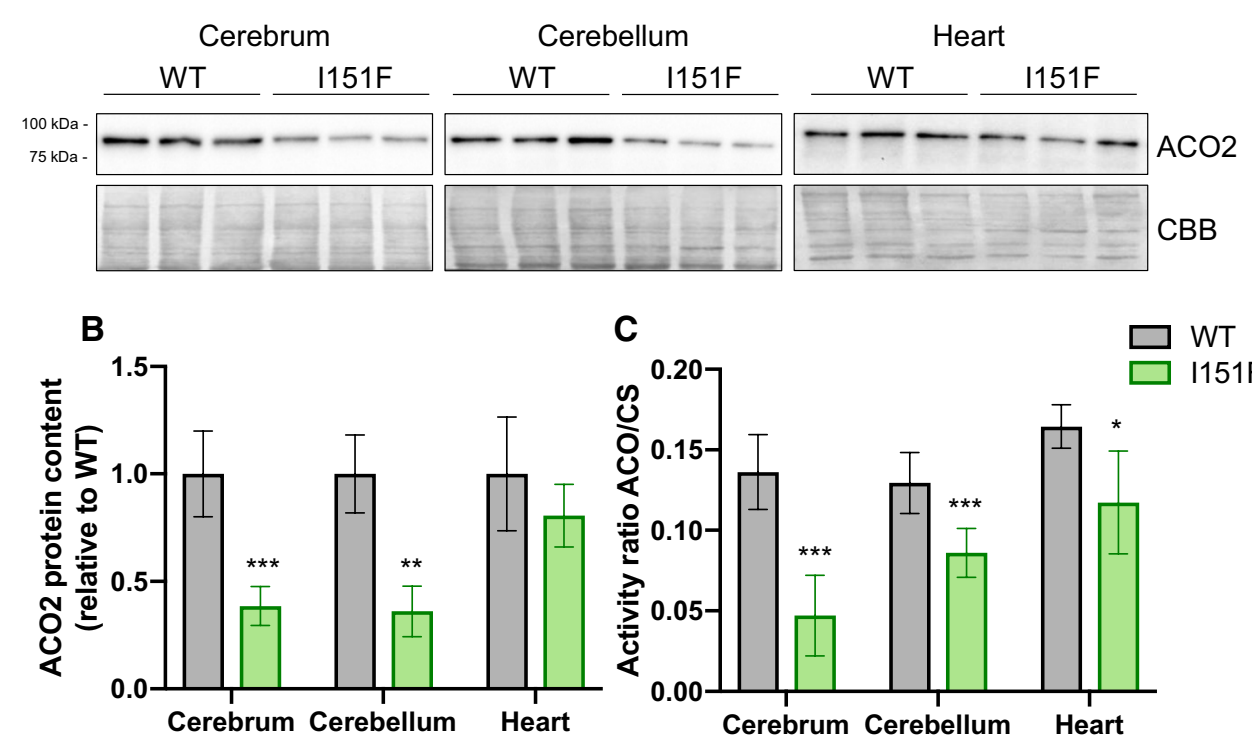

D

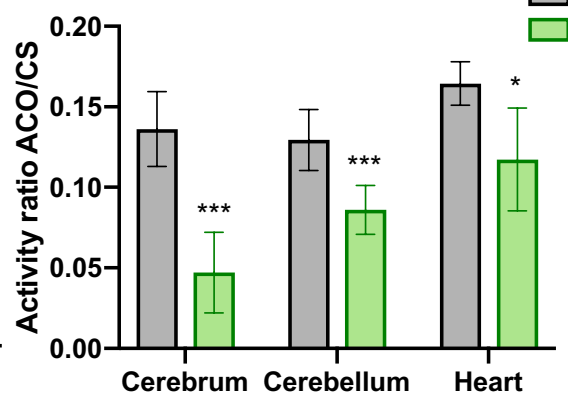

39 weeks
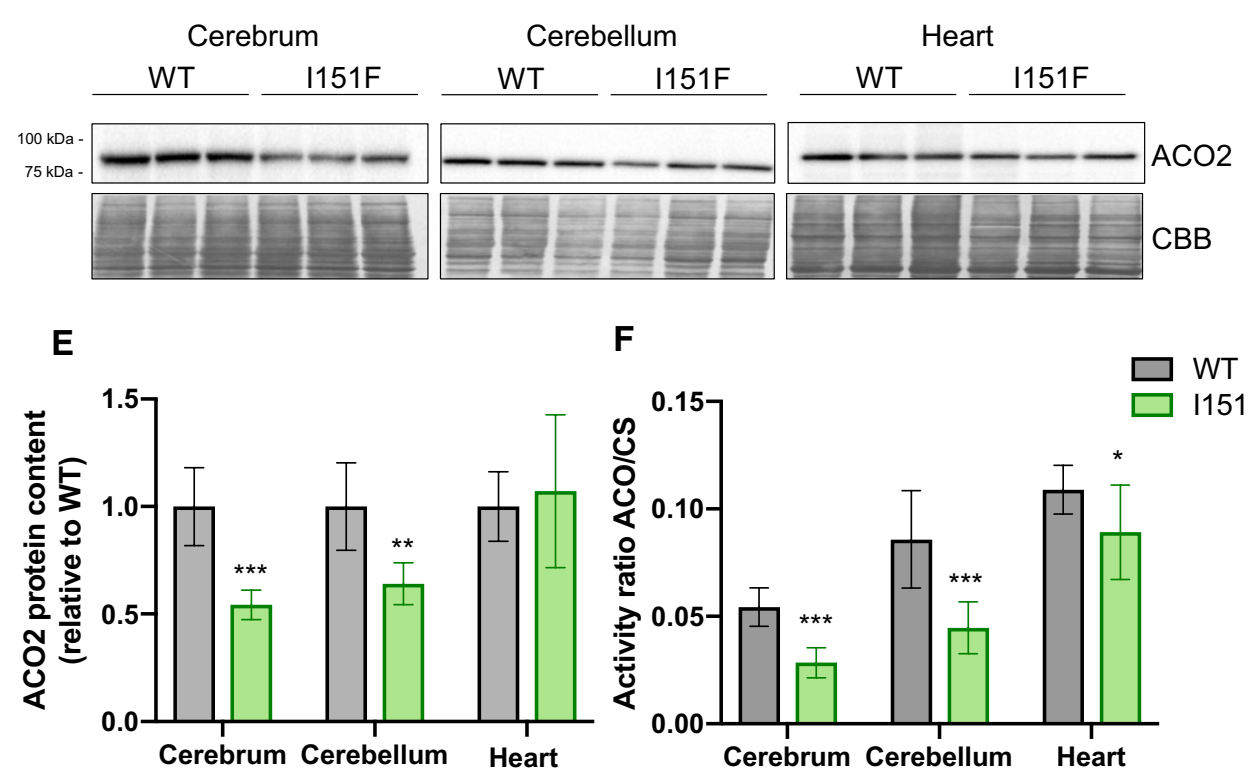

\section{mRNA levels from NDUFB8, SDHA, SDHB, and ACO2}

In order to discern whether the decreased content of NDUFB8, SDHA, SDHB, and ACO2 proteins was caused by lowered gene expression or by other mechanisms, the mRNA levels of these genes were analyzed by qPCR (Figure 8). We performed this analysis in cerebellum and heart tissues from 21- and 39-week-old mice. In cerebellum, no changes were found in the mRNA levels of these genes when comparing WT and $\mathrm{FXN}^{\mathrm{I} 151 \mathrm{~F}}$ mice. This result indicates that changes in protein content in this tissue are caused by posttranscriptional mechanisms. Regarding heart, a decrease in SDHB and NDUFB8 mRNA levels was observed in 21-week-old animals. Therefore, the decreased content of these proteins in heart can be attributed to a lowered gene expression. Indeed, in 39-week-old mice, the mRNA levels of both proteins are restored, as observed from protein content. In contrast, SDHA expression is not altered in 21-week-old mouse heart (despite lower protein content). Therefore, loss of SDHA in heart is also caused by posttranscriptional mechanisms. Overall, these results suggest that the mechanisms causing alterations in protein content may be tissue specific, and include transcriptional and posttranscriptional mechanisms. 
A

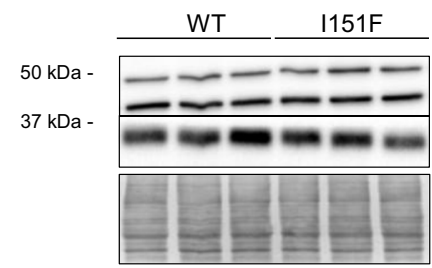

$25 \mathrm{kDa}$ $20 \mathrm{kDa}$

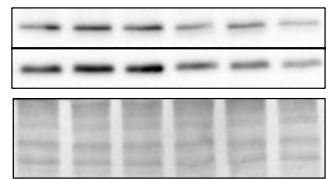

B

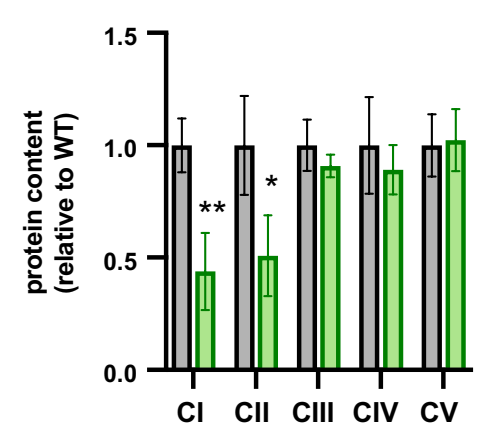

C

Cerebrum

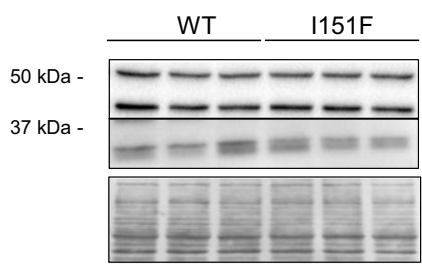

$25 \mathrm{kDa}$ -

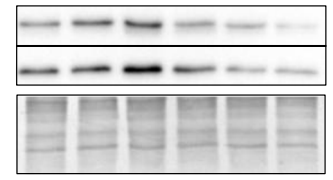

D

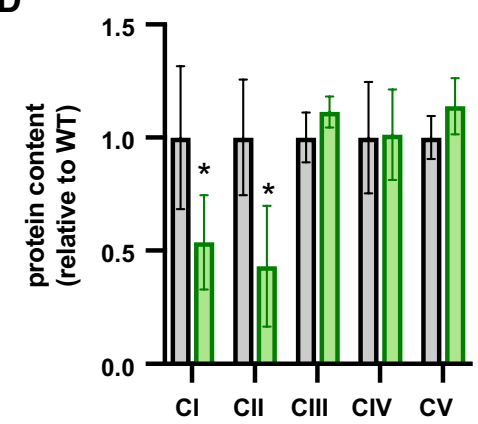

\section{1 weeks}

Cerebellum
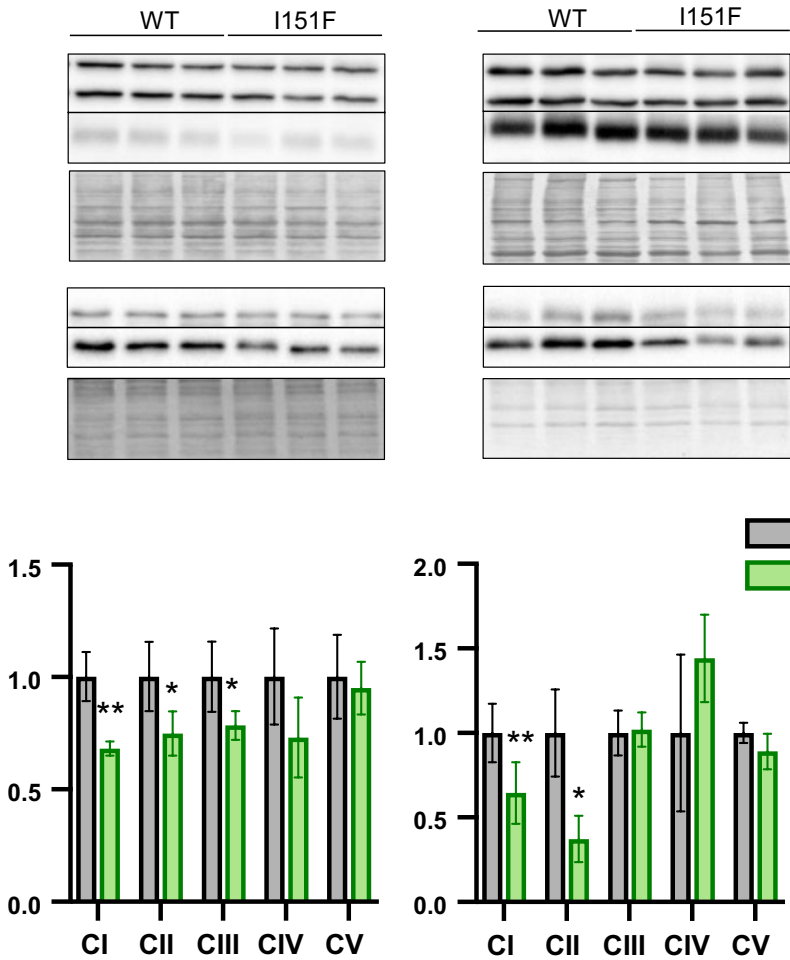

Heart
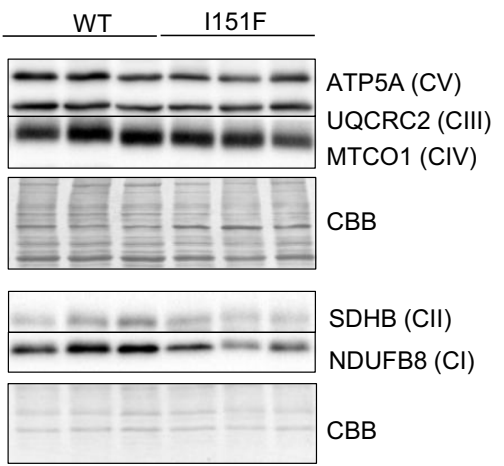

WT I151F

39 weeks

Cerebellum

Heart
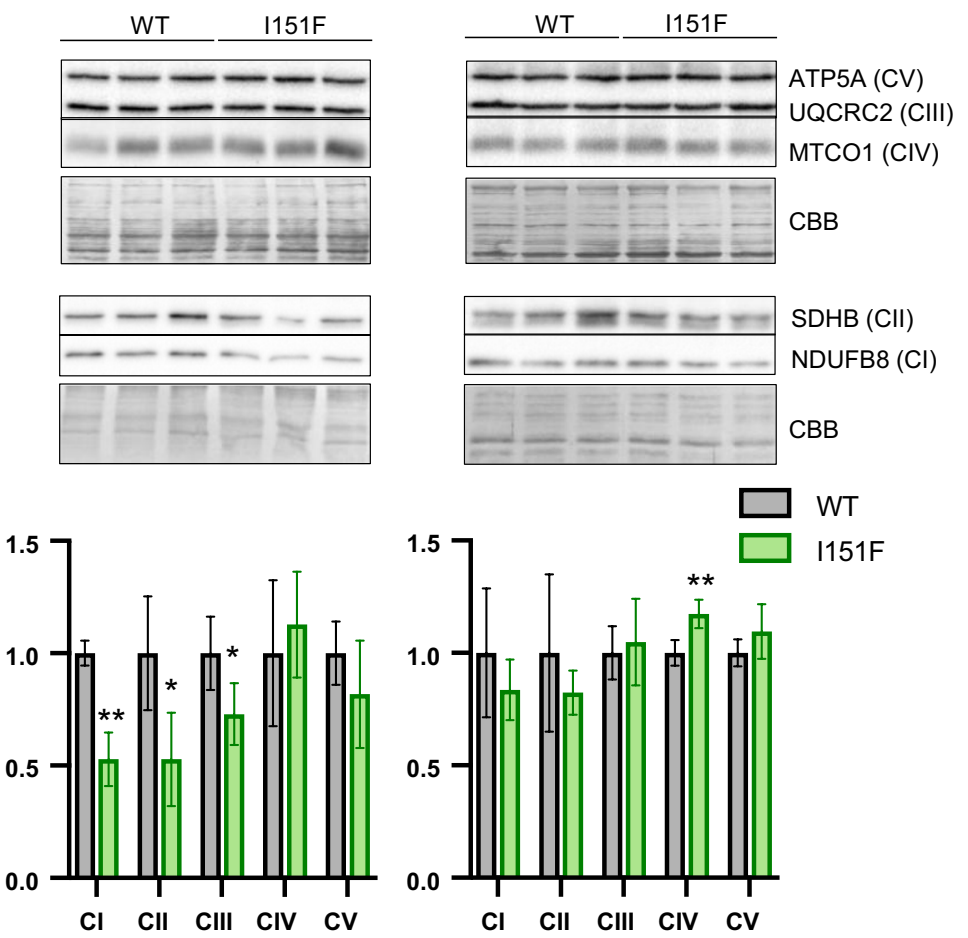
4Fig. 7 Western blot analysis of components of the OXPHOS system. The components indicated of the mitochondrial OXPHOS system were analyzed by western blot in cerebrum, cerebellum, and heart homogenates from 21-week (A) and 39-week (C) WT and $\mathrm{FXN}^{\mathrm{I1} 151 \mathrm{~F}}$ mice. Representative western blot images are shown in $\mathbf{A}$ and $\mathbf{C}$. Samples from three different mice were loaded on gels. Histograms $\mathbf{B}$ and $\mathbf{D}$ represent the mean \pm SD from at least four independent measurements. Significant differences between $\mathrm{WT}$ and $\mathrm{FXN}^{\mathrm{I15} 1 \mathrm{~F}}$ mice are indicated (p values $<0.05(*), 0.01(* *)$, or $0.001(* * *)$

\section{Biosynthesis of lipoic acid is not compromised in FXN ${ }^{1151 F}$ mice}

We also decided to analyze the content of protein-bound lipoic acid, a prosthetic group required for PDH activity. This analysis had two objectives: first, to complement the results from the targeted proteomics analysis, where several components of the PDH complex were analyzed; second, to further analyze the status of iron-sulfur clusters in $\mathrm{FXN}^{\mathrm{I} 151 \mathrm{~F}}$ mice, as synthesis of this cofactor requires lipoate synthase, which is an iron-sulfur containing enzyme. Western blot analysis of cerebrum, cerebellum, and heart lysates using antibodies raised against lipoate revealed the presence of two bands, at 70 and $50 \mathrm{kDa}$ apparent molecular weight (Figure 9). The $70 \mathrm{kDa}$ band (which is more intense) corresponds to lipoic acid bound to DLAT (the E2 component of $\mathrm{PDH}$ ), while three lipoic acid containing proteins migrate at $50 \mathrm{kDa}$. These are the E2 component of alpha-ketoglutarate dehydrogenase (Dihydrolipoyllysine succinyltransferase, DLST), the E2 component of the branched chain alphaketodehydrogenase complex, and the PDH-binding component X (a structural subunit of the PDH complex) [22]. As indicated in Figure 9, no significant differences were observed in DLAT-bound lipoic acid (70 kDa) between WT and $\mathrm{FXN}^{\mathrm{I1} 151 \mathrm{~F}}$ mice. Decreased content in the $50 \mathrm{kDa}$ band was observed in 21-week-old cerebrum, but not in the other tissues analyzed. This band was also decreased in 39-weekold mice, although here differences did not reach statistical significance. Overall, these results indicate that proteinbound lipoic acid biosynthesis (which requires an iron-sulfur enzyme) is not compromised in $\mathrm{FXN}^{\mathrm{I}}{ }^{151 \mathrm{~F}}$ mice.

\section{Discussion}

In the present work we have analyzed the consequences of the FXN I151F mutation in mice, which is equivalent to the human I154F pathological mutation. This mutation was shown to cause partial loss of function [26, 27], decreased frataxin stability, and also accumulation of insoluble frataxin proteoforms [25]. This last observation suggested that these insoluble forms could be involved in the pathological mechanism of the $1154 \mathrm{~F}$ mutation. From our in vivo results, we can conclude that the primary biochemical consequence of this mutation is decreased frataxin content, as we have observed that $\mathrm{FXN}^{\mathrm{I} 151 \mathrm{~F}}$ homozygous mice present very low frataxin levels in all tissues analyzed. In contrast, we dit not observe the presence of the insoluble frataxin proteoforms reported by $\mathrm{Li}$ and collaborators in cells overexpressing human FXN I154 [25]. As we have used similar protein extraction methods to those of the referenced work, we hypothesize that the presence of such insoluble intermediate forms in vitro was likely the consequence of frataxin overexpression under non-physiological conditions. Moreover, we observed that $\mathrm{FXN}^{\mathrm{I} 151 \mathrm{~F}}$ mice present severe phenotypes which resemble those seen in other FA models. First, they present decreased weight gain, which is observed from 15 weeks onward. Second, they present neurological deficits, which manifest from week 23 onward. Third, they present marked biochemical alterations, which are observed before the appearance of the functional alterations (they are already seen in 21-week-old mice).

The behavioral tasks performed indicate that $\mathrm{FXN}^{\mathrm{I} 151 \mathrm{~F}}$ mice exhibit several neurological deficits that are reminiscent of those observed in FA patients. Thus, $\mathrm{FXN}^{\mathrm{I}}{ }^{51 \mathrm{~F}}$ mice displayed decreased locomotor activity, defects in their forelimb muscular strength, reduced motor coordination and balance, and reduced hind and front limb stride length when compared with WT controls. Also, the results obtained indicate that neurological defects are progressive, as they are not manifested until 22 weeks of age. Indeed, most of them are observed at older ages. These defects may be caused by degeneration of dorsal root ganglia and of the dentate nucleus of the cerebellum, as observed in FA patients.

An interesting observation from this work is that biochemical alterations are observed before the onset of the functional disturbances, and that these alterations are not progressive in the nervous system (they are similar in 21and 39-week-old mice). Among the proteins analyzed, we found decreased content of NDUFB8 (from complex I), SDHA and SDHB (from complex II), and ACO2. This observation is consistent with previous results in the KIKO mouse model, where complex I and II enzyme activities were also decreased in cerebella from asymptomatic mice [19]. Gene expression analysis indicates that loss of these proteins in cerebellum is caused by posttranscriptional events. As NDUF8, SDHA, SDHB, and ACO2 have in common that they either contain iron-sulfur clusters (SDHB and ACO2) or belong to complexes containing them (complex I and II), it could be hypothesized that these proteins are degraded due to the absence of this cofactor. Nevertheless, we should be cautious about this hypothesis, because $\mathrm{FXN}^{\mathrm{I151F}}$ mice do not present a general loss of iron-sulfur clusters. This is evidenced by the high aconitase content and activity found in heart, and also by the normal presence of protein-bound lipoate under most of the conditions analyzed (protein-bound lipoate 
Fig. 8 Analysis of mRNA expression by qPCR. Histograms show the relative mRNA content of the genes indicated in cerebellum $(\mathbf{A})$ or heart $(\mathbf{B})$ from 21- and 39-week-old WT and $\mathrm{FXN}^{\mathrm{I151F}}$ mice. Data are represented as mean \pm SD from six mice. Significant differences p values $<0.05(*), 0.01(* *)$, or $0.001(* * *)$ between WT and $\mathrm{FXN}^{\mathrm{I} 151 \mathrm{~F}}$ mice are indicated
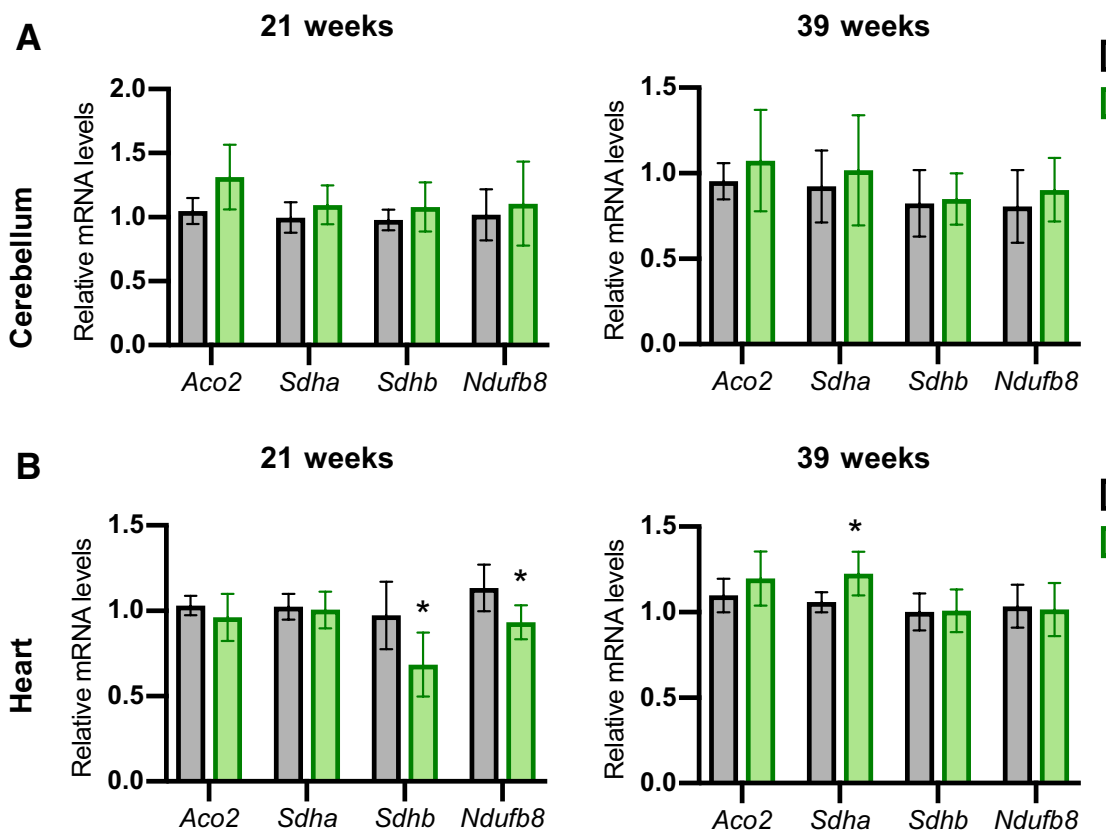

WT I151F

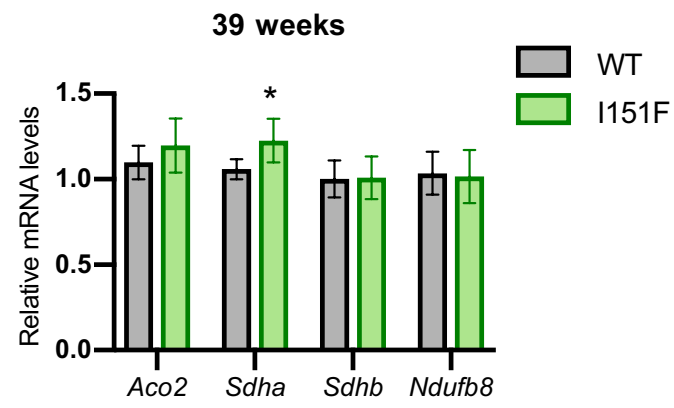

requires an iron-sulfur containing protein for its biosynthesis [35]). It is worth reminding that iron-sulfur loss is not a universal characteristic of frataxin deficiency [6, 36]. Therefore, the mechanism explaining the loss of these proteins in $\mathrm{FXN}^{\mathrm{I} 151 \mathrm{~F}}$ mice may involve different regulatory pathways at either transcriptional or posttranscriptional levels. In this regard, ACO2, NDUF8, and SDHB are known to be regulated by posttranscriptional mechanisms. Studies in frataxin deficient yeasts indicate that aconitase and SDHB deficiency are caused by $\mathrm{Cth} 2$, an mRNA binding protein that regulates the expression of iron-containing proteins at the posttranscriptional level [37]. In mammals, ACO2, NDUF8, and SDHB are downregulated in conditions of iron deficiency [38], but the mechanisms causing this decrease are not the same for all of them. ACO2 downregulation is caused by the presence of an iron responsive element (IRE) in its mRNA, 5' from the coding region. Upon iron starvation, Iron regulatory proteins (IRPs) bind to this site and block mRNA translation [39]. In contrast, NDUF8 and SDHB are not regulated by IRPs, as their mRNAs do not contain IREs. The existence of several regulatory mechanisms may explain why different mRNA and protein expression changes are observed in cerebellum and heart from $\mathrm{FXN}^{\mathrm{I151F}}$ mice. In heart, changes in SDHB and NDUF8 content were observed both at the mRNA and protein level, while in cerebellum we observe decreased protein content with no changes in mRNA levels. These observations indicate that the mechanisms may be tissue specific and must include transcriptional and posttranscriptional events. Thus, a detailed focus on these events will be required to elucidate the mechanisms causing changes in the proteome of
FXN $^{151 F}$ mice. Actually, IRP activation has been described in FA models [6], therefore, IRP activation could be one of these mechanisms.

The targeted proteomic analysis also revealed changes in SOD1 and SOD2 protein content. These alterations are more marked in heart than in the nervous system. Both enzymes are involved in superoxide scavenging and had been previously described as either induced or decreased in frataxin-deficient cells or mice [31, 40-42]. Interestingly, we observed both phenomena, as both enzymes are induced in 21-week-old mice, while their content is decreased in 39-week-old mice. The mechanism causing this paradoxical behavior could be complex, as several transcription factors are described to regulate superoxide dismutase expression. In this regard, the promoter of the SOD1 gene contains binding sites for at least seven transcription factors, and at least two of these, NRF2 and $\mathrm{NF}-\kappa \mathrm{B}$, could be responding to the oxidative stress conditions caused by frataxin deficiency [43]. Moreover, it has also been described that NRF2 signaling may be inhibited in frataxin-deficient cells [13]14. Thus, SOD expression would depend on the balance between activation of these transcription factors by stress, and inhibition of NRF2 signaling. Such balance could be different at different ages, explaining the differences observed between 21- and 39-week-old mice. It is worth commenting that a similar behavior was seen in hearts from the cardiac KO mouse model (MCK/frataxin), which showed a modest SOD2 induction between two and seven weeks followed by a dramatic decrease at later ages [7]. Another potential explanation for the SODs decline observed in 39-week-old hearts could be related to alterations in metal homeostasis caused by frataxin deficiency. In this regard, studies in yeast showed that the activities of both SOD1 and 
Fig. 9 Western blot analysis of protein-bound lipoic acid. Protein bound lipoic acid was analyzed using lipoic acid (LA) antibodies in cerebrum, cerebellum and heart homogenates from 21-week (A) and 39-week (C) WT and FXN ${ }^{\mathrm{I} 151 \mathrm{~F}}$ mice. Representative western blot images are shown in $\mathbf{A}$ and C. Samples from three different mice were loaded on gels. The $70 \mathrm{kDa}$ band corresponds to lipoic acid bound to DLAT, while three lipoic acid containing proteins migrate at $50 \mathrm{kDa}$ (DLST, E2 from the branched chain alpha-ketodehydrogenase complex, and PDH-X). Histograms (B and $\mathbf{D})$ represent the mean value \pm SD from each band calculated from four independent measurements. Significant differences between WT and $\mathrm{FXN}^{\mathrm{I} 151 \mathrm{~F}}$ mice are indicated $(\mathrm{p}$ value $<0.01(* *))$

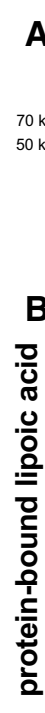

A

21 weeks
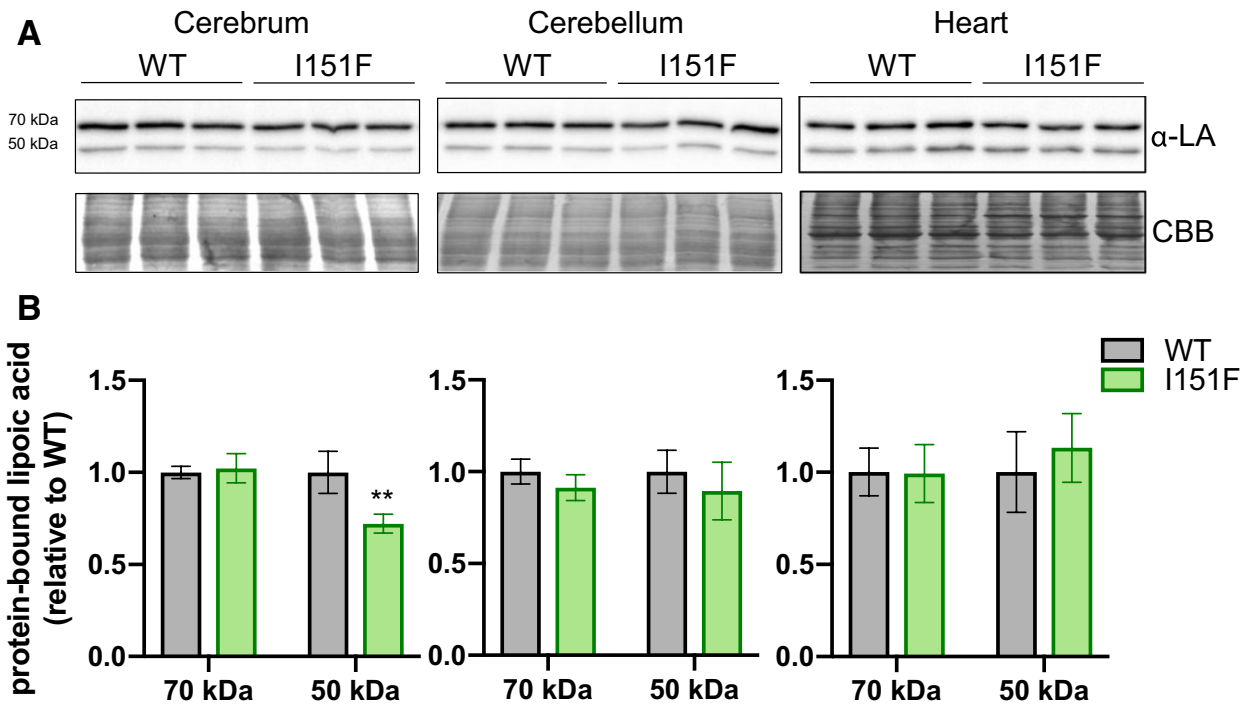

39 weeks
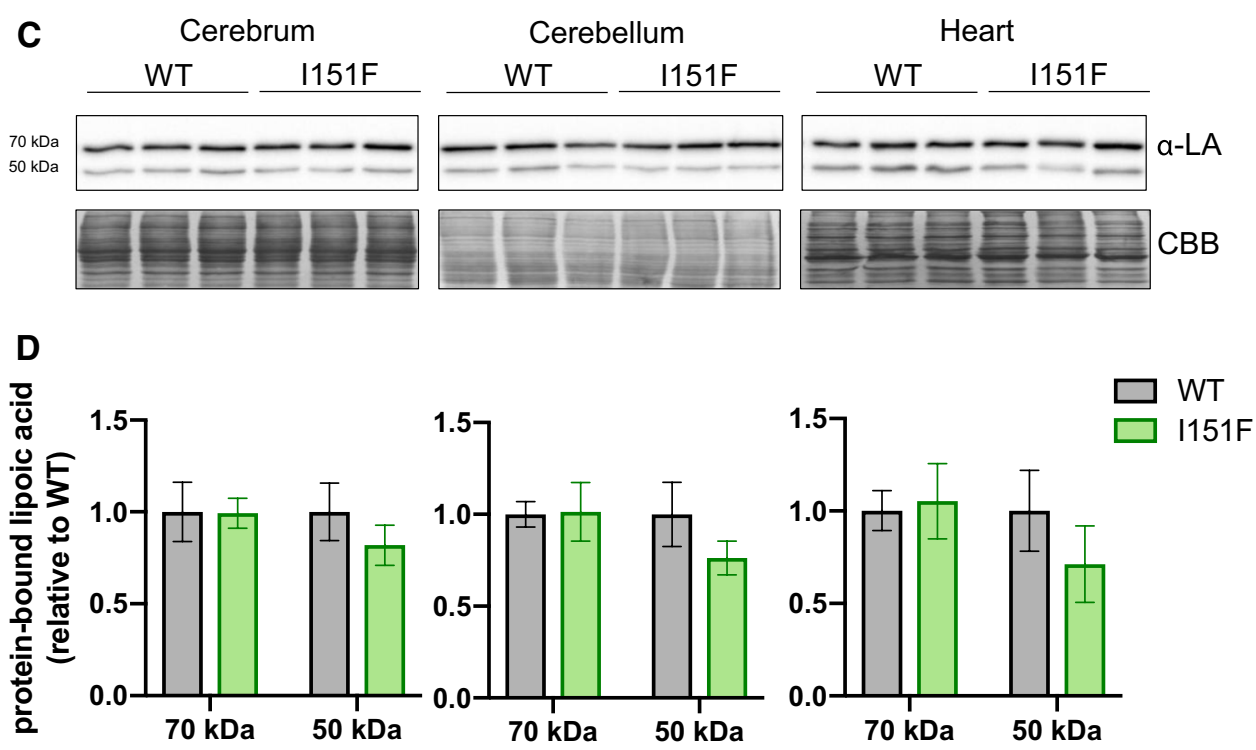

SOD2 were compromised due to limited availability of $\mathrm{Cu}$ and $\mathrm{Mn}$, their metal cofactors. This limited $\mathrm{Cu}$ and $\mathrm{Mn}$ availability would be caused by interferences related to disrupted iron homeostasis [44].

In this work we also analyzed the content of three PDH containing components. We had previously observed decreased PDHA1 content and disturbances in the redox state of DLAT-bound lipoic acid in primary cultures of frataxin-deficient neonatal rat ventricular cardiomyocytes (NRVMs) [22]. These previous results indicated a $40 \%$ loss of PDHA1 content, and no major changes in DLAT or DLDH content. Indeed, treatment with the PDH activator dichloroacetate restored some of the defects found in frataxin-deficient NRVM [22]. The results obtained in the present work indicate a slight PDHA1 decrease in 21-week-old mice, while this protein was induced in hearts from 39-week-old mice. Regarding DLDH, we observed a 25 to $30 \%$ content loss in hearts and cerebellum from 39-week-old $\mathrm{FXN}^{\mathrm{I1} 151 \mathrm{~F}}$ mice. In this context, the previous results in rat cardiomyocytes indicated that DLAT-bound lipoic acid was more oxidized in frataxin-deficient cells, and the decreased DLDH content in $\mathrm{FXN}^{\mathrm{I} 151 \mathrm{~F}}$ mice could cause a similar consequence. Therefore, we have observed alterations in the PDH complex, although the physiological consequences of these subtle differences, if any, would require further research.

Finally, the results from this work also indicate that the $\mathrm{FXN}^{\mathrm{I} 151 \mathrm{~F}}$ mouse can be an excellent tool for analyzing the 
consequences of frataxin deficiency and for testing new therapies. Generating mouse models to recreate FA has been challenging, as this disease manifest when frataxin levels are below a pathological threshold. Therefore, animals must express some frataxin to be viable, but such expression may be lower than the pathological threshold required to trigger the disease. Actually, several FA mouse models have been generated, which can be classified into four main categories: 1) tissue specific KOs, which present a total loss of frataxin in some tissues; 2) GAA-repeat mice models, which harbor a GAA expansion in the frataxin gene; 3) an inducible mutant, in which frataxin expression is repressed by a shRNA transgene placed under the control of a TET promoter (doxycycline-inducible) [45]; 4) point mutation models, in which mouse frataxin has been genetically modified by CRISPR to present a pathological point mutation. All these models present limitations that could be overcome by the FXN ${ }^{\mathrm{I} 151 \mathrm{~F}}$ model. Thus, tissue-specific KO mice [46] can be used to analyze the specific consequences of frataxin deficiency in a specific tissue, but they do not mimic the biochemical problem encountered by patients (the presence of limited amounts of frataxin in all tissues). GAA-repeat mice models have the advantage of mimicking the most common mutation found in patients (the GAA expansion). Nevertheless, all the GAA-repeat models developed to date present a mild phenotype, suggesting that the frataxin expression in these models is above (or slightly below) the pathological threshold required to trigger FA-like phenotypes in mice (reviewed in [47, 48]. This limits its usefulness as a model to study the pathophysiology of the disease and also to test new therapeutic approaches, as no clear biomarkers of disease severity can be defined. Regarding models based on point mutations, a partial characterization of a G127V FXN mouse model (equivalent to human pathological mutation G130V) was recently published. This model has a severe phenotype, as mice harboring this mutation presented a substantially reduced number of offspring. Mouse embryonic fibroblasts derived from this model exhibit significantly reduced proliferation and bioenergetic alterations [49], but there is no published data about the consequences of this mutation in adult mice. Therefore, the $\mathrm{FXN}^{\mathrm{I} 151 \mathrm{~F}}$ mouse model is the only FA model which presents decreased frataxin content in all tissues and clear biomarkers. These features make this model an excellent tool for the study of FA pathophysiology and also for testing therapeutic approaches focused on frataxin replacement (i.e., gene therapy) or on preventing the consequences of frataxin deficiency. On the other hand, it is not suitable for testing several therapeutic candidates, like those drugs focused on increasing endogenous frataxin expression.

In summary, the results obtained indicate that the pathological mechanisms of the FXN I154F mutation are triggered by decreased frataxin content as i) decreased content of frataxin is found in all tissues analyzed, ii) the presence of insoluble intermediate forms was not observed, and iii) the phenotypes observed resemble those seen in FA patients and models. These observations are consistent with clinical data, as the clinical phenotype of patients carrying the $\mathrm{I} 154 \mathrm{~F}$ mutation (compound heterozygotes for the GAA tripletrepeat expansion and the point mutation) is similar to that of individuals homozygous for the GAA expansion [24]. Therefore, patients carrying this mutation would benefit from gene therapy or other frataxin replacement therapies. Our results also indicate that the $\mathrm{FXN}^{\mathrm{I} 151 \mathrm{~F}}$ mouse is an excellent model for FA research, which can be used for investigating the pathological consequences of frataxin deficiency. In this regard, the presence of clear biochemical and functional biomarkers indicates that this model can be used to test the effect of several therapeutic approaches.

Supplementary Information The online version contains supplementary material available at https://doi.org/10.1007/s00018-021-04100-5.

Acknowledgements The authors would like to thank Aamir Zubery and Cathleen Lutz from the Rare and Orphan Disease Center at the Jackson Laboratory for $\mathrm{FXN}^{\mathrm{I} 151 \mathrm{~F}}$ HET mice model generation. We thank Roser Pané for technical assistance, as well as the Proteomics and the Animal services of the Universitat de Lleida, and the metabolomic services of the IRBLLeida. We also thank Dr Esther Vilaprinyó (Universitat de Lleida) for advice in statistical analysis.

Authors' contributions MMC and ASA: performed most of the experiments and analyzed the data. EB and FD: assisted in the collection and preparation of mouse tissues. EC: assisted in the biochemical analyses. All authors provided technical support and suggestions for the project and for the manuscript. JR and JT: conceived the project and supervised the study. MMC and JT: designed the experiments, analyzed and interpreted data, and wrote the manuscript.

Funding Open Access funding provided thanks to the CRUE-CSIC agreement with Springer Nature. FXN ${ }^{\mathrm{I} 151 \mathrm{~F}}$ Het mice were generated thanks to the BeHeard Challenge hosted by the Rare Genomics Institute (CA, USA). This work has also been funded by grants from Association Française de l'Ataxie de Friedreich - AFAF (obtained by FD) and from Ministerio de Economía y Empresa (MINECO, Spain, SAF201783883-R) (obtained by JR and JT).

Availability of data and material Mass spectrometry data produced in this study is available in Panorama Public. https://panoramaweb.org/ FxnI151FMs.url

\section{Declarations}

Conflict of interests The authors declare that they have no conflict of interest.

Ethics approval The investigation with experimental animals conforms to the National Guidelines for the regulation of the use of experimental laboratory animals from the Generalitat de Catalunya and the Government of Spain (article 33.a 214/1997) and was evaluated and approved by the Experimental Animal Ethical Committee of the Universitat de Lleida (CEEA).

Consent for publication Not applicable. 
Open Access This article is licensed under a Creative Commons Attribution 4.0 International License, which permits use, sharing, adaptation, distribution and reproduction in any medium or format, as long as you give appropriate credit to the original author(s) and the source, provide a link to the Creative Commons licence, and indicate if changes were made. The images or other third party material in this article are included in the article's Creative Commons licence, unless indicated otherwise in a credit line to the material. If material is not included in the article's Creative Commons licence and your intended use is not permitted by statutory regulation or exceeds the permitted use, you will need to obtain permission directly from the copyright holder. To view a copy of this licence, visit http://creativecommons.org/licenses/by/4.0/.

\section{References}

1. Parkinson MH, Boesch S, Nachbauer W et al (2013) Clinical features of Friedreich's ataxia: classical and atypical phenotypes. J Neurochem 126(Suppl):103-117. https://doi.org/10.1111/jnc. 12317

2. Tsou AY, Paulsen EK, Lagedrost SJ et al (2011) Mortality in Friedreich ataxia. J Neurol Sci 307:46-49. https://doi.org/10.1016/j. jns.2011.05.023

3. Campuzano V, Montermini L, Molto MD, et al (1996) Friedreich's ataxia: autosomal recessive disease caused by an intronic GAA triplet repeat expansion. Science (80- ) 271:1423-1427

4. Galea CA, Huq A, Lockhart PJ et al (2016) Compound heterozygous $F X N$ mutations and clinical outcome in friedreich ataxia. Ann Neurol 79:485-495. https://doi.org/10.1002/ana.24595

5. Gervason S, Larkem D, Ben MA et al (2019) Physiologically relevant reconstitution of iron-sulfur cluster biosynthesis uncovers persulfide-processing functions of ferredoxin- 2 and frataxin. Nat Commun 10:3566. https://doi.org/10.1038/s41467-019-11470-9

6. Alsina D, Purroy R, Ros J, Tamarit J (2018) Iron in Friedreich Ataxia: A Central Role in the Pathophysiology or an Epiphenomenon? Pharmaceuticals 11:89. https://doi.org/10.3390/ph11030089

7. Seznec H, Simon D, Bouton C et al (2005) Friedreich ataxia: the oxidative stress paradox. Hum Mol Genet 14:463-474

8. Li K, Besse EK, Ha D et al (2008) Iron-dependent regulation of frataxin expression: implications for treatment of Friedreich ataxia. Hum Mol Genet 17:2265-2273

9. Whitnall M, Rahmanto YS, Sutak R et al (2008) The MCK mouse heart model of Friedreich's ataxia: Alterations in iron-regulated proteins and cardiac hypertrophy are limited by iron chelation. Proc Natl Acad Sci U S A 105:9757-9762

10. Gakh O, Park S, Liu G et al (2006) Mitochondrial iron detoxification is a primary function of frataxin that limits oxidative damage and preserves cell longevity. Hum Mol Genet 15:467-479. https:// doi.org/10.1093/hmg/ddi461

11. Tamarit J, Britti E, Delaspre F, et al (2021) Crosstalk between nucleus and mitochondria in human disease: Mitochondrial iron and calcium homeostasis in Friedreich ataxia. 73:543-553 IUBMB Life. https://doi.org/10.1002/iub.2457

12. Condò I, Malisan F, Guccini I et al (2010) Molecular control of the cytosolic aconitase/IRP1 switch by extramitochondrial frataxin. Hum Mol Genet 19:1221-1229. https://doi.org/10.1093/ hmg/ddp592

13. Paupe V, Dassa EP, Goncalves $S$ et al (2009) Impaired nuclear Nrf2 translocation undermines the oxidative stress response in Friedreich ataxia. PLoS ONE 4:e4253. https://doi.org/10.1371/ journal.pone.0004253

14. Shan Y, Schoenfeld RA, Hayashi G et al (2013) Frataxin deficiency leads to defects in expression of antioxidants and Nrf2 expression in dorsal root ganglia of the Friedreich's ataxia YG8R mouse model. Antioxid Redox Signal 19:1481-1493. https://doi. org/10.1089/ars.2012.4537

15. Cotticelli MG, Xia S, Lin D, et al (2019) Ferroptosis as a novel therapeutic target for Friedreich's ataxia. J Pharmacol Exp Ther jpet.118.252759. https://doi.org/10.1124/jpet.118.252759

16. Du J, Zhou Y, Li Y et al (2020) Identification of Frataxin as a regulator of ferroptosis. Redox Biol 32:101483. https://doi.org/ 10.1016/j.redox.2020.101483

17. Jiang X, Stockwell BR, Conrad M (2021) Ferroptosis: mechanisms, biology and role in disease. Nat Rev Mol Cell Biol 22:266282. https://doi.org/10.1038/s41580-020-00324-8

18. Tamarit J, Obis Ė, Ros J (2016) Oxidative stress and altered lipid metabolism in Friedreich ataxia. Free Radic Biol Med 100:138-146. https://doi.org/10.1016/j.freeradbiomed.2016. 06.007

19. Lin H, Magrane J, Rattelle A et al (2017) Early cerebellar deficits in mitochondrial biogenesis and respiratory chain complexes in the KIKO mouse model of Friedreich ataxia. Dis Model Mech 10:1343-1352. https://doi.org/10.1242/dmm.030502

20. Britti E, Delaspre F, Sanz-Alcázar A et al (2021) Calcitriol increases frataxin levels and restores mitochondrial function in cell models of Friedreich Ataxia. Biochem J 478:1-20. https:// doi.org/10.1042/BCJ20200331

21. Purroy R, Britti E, Delaspre F et al (2018) Mitochondrial pore opening and loss of $\mathrm{Ca} 2+$ exchanger NCLX levels occur after frataxin depletion. Biochim Biophys Acta - Mol Basis Dis 1864:618-631. https://doi.org/10.1016/J.BBADIS.2017.12.005

22. Purroy R, Medina-Carbonero M, Ros J, Tamarit J (2020) Frataxindeficient cardiomyocytes present an altered thiol-redox state which targets actin and pyruvate dehydrogenase. Redox Biol 32:101520. https://doi.org/10.1016/j.redox.2020.101520

23. Rodríguez LR, Calap-Quintana P, Lapeña-Luzón T et al (2020) Oxidative stress modulates rearrangement of endoplasmic reticulum-mitochondria contacts and calcium dysregulation in a Friedreich's ataxia model. Redox Biol 37:101762. https://doi.org/10. 1016/J.REDOX.2020.101762

24. Galea CA, Huq A, Lockhart PJ et al (2015) Compound heterozygous FXN mutations and clinical outcome in Friedreich ataxia. Ann Neurol 79:485-495. https://doi.org/10.1002/ana.24595

25. Li H, Gakh O, Smith DY et al (2013) Missense mutations linked to Friedreich ataxia have different but synergistic effects on mitochondrial frataxin isoforms. J Biol Chem 288:4116-4127. https:// doi.org/10.1074/jbc.M112.435263

26. Shan Y, Napoli E, Cortopassi G (2007) Mitochondrial frataxin interacts with ISD11 of the NFS1/ISCU complex and multiple mitochondrial chaperones. Hum Mol Genet 16:929-941. https:// doi.org/10.1093/hmg/ddm038

27. Tsai C-L, Bridwell-Rabb J, Barondeau DP (2011) Friedreich's Ataxia Variants I154F and W155R Diminish Frataxin-Based Activation of the Iron-Sulfur Cluster Assembly Complex. Biochemistry 50:6478-6487. https://doi.org/10.1021/bi200666h

28. Gessulat S, Schmidt T, Zolg DP et al (2019) Prosit: proteome-wide prediction of peptide tandem mass spectra by deep learning. Nat Methods 16:509-518. https://doi.org/10.1038/s41592-019-0426-7

29. MacLean B, Tomazela DM, Shulman N et al (2010) Skyline: an open source document editor for creating and analyzing targeted proteomics experiments. Bioinformatics 26:966-968. https://doi. org/10.1093/bioinformatics/btq054

30. Rotig A, de Lonlay P, Chretien D et al (1997) Aconitase and mitochondrial iron-sulphur protein deficiency in Friedreich ataxia. Nat Genet 17:215-217

31. Irazusta V, Cabiscol E, Reverter-Branchat $\mathrm{G}$ et al (2006) Manganese is the link between frataxin and iron-sulfur deficiency in the yeast model of Friedreich ataxia. J Biol Chem 281:12227-12232. https://doi.org/10.1074/jbc.M511649200 
32. Patraa S, Barondeaua DP (2019) Mechanism of activation of the human cysteine desulfurase complex by frataxin. Proc Natl Acad Sci U S A 116:19421-19430. https://doi.org/10.1073/pnas.19095 35116

33. Gonzalez-Cabo P, Vazquez-Manrique RP, Garcia-Gimeno MA et al (2005) Frataxin interacts functionally with mitochondrial electron transport chain proteins. Hum Mol Genet 14:2091-2098

34. Dong YN, McMillan E, Clark EM et al (2019) GRP75 overexpression rescues frataxin deficiency and mitochondrial phenotypes in Friedreich ataxia cellular models. Hum Mol Genet 28:1594-1607. https://doi.org/10.1093/hmg/ddy448

35. Ollagnier-de Choudens S, Sanakis Y, Hewitson KS et al (2000) Iron-sulfur center of biotin synthase and lipoate synthase. Biochemistry 39:4165-4173. https://doi.org/10.1021/bi992090u

36. Bayot A, Santos R, Camadro JM, Rustin P Friedreich's ataxia: the vicious circle hypothesis revisited. BMC Med. 9:112-119. https:// doi.org/10.1186/1741-7015-9-112

37. Moreno-Cermeno A, Alsina D, Cabiscol E et al (2013) Metabolic remodeling in frataxin-deficient yeast is mediated by $\mathrm{Cth} 2$ and Adr1. Biochim Biophys Acta 1833:3326-3337. https://doi.org/ 10.1016/j.bbamcr.2013.09.019

38. Leermakers PA, Remels AHV, Zonneveld MI et al (2020) Iron deficiency-induced loss of skeletal muscle mitochondrial proteins and respiratory capacity; the role of mitophagy and secretion of mitochondria-containing vesicles. FASEB J 34:6703-6717. https://doi.org/10.1096/fj.201901815R

39. Sanchez M, Galy B, Schwanhaeusser B et al (2011) Iron regulatory protein-1 and -2: transcriptome-wide definition of binding mRNAs and shaping of the cellular proteome by iron regulatory proteins. Blood 118:168-179. https://doi.org/10.1182/ blood-2011-04-343541

40. Santos MM, Ohshima K, Pandolfo M (2001) Frataxin deficiency enhances apoptosis in cells differentiating into neuroectoderm. Hum Mol Genet 10:1935-1944

41. Sturm B, Bistrich U, Schranzhofer M et al (2005) Friedreich's Ataxia : no changes in mitochondrial labile iron in human lymphoblasts and fibroblasts. A decrease in antioxidative capacity? J Biol Chem 280:6701-6708. https://doi.org/10.1074/jbc.M408717200
42. Chantrel-Groussard K, Geromel V, Puccio H et al (2001) Disabled early recruitment of antioxidant defenses in Friedreich's ataxia. Hum Mol Genet 10:2061-2067

43. Milani P, Gagliardi S, Cova E, Cereda C (2011) SOD1 Transcriptional and Posttranscriptional Regulation and Its Potential Implications in ALS. Neurol Res Int 2011:1-9. https://doi.org/10.1155/ $2011 / 458427$

44. Irazusta V, Obis E, Moreno-Cermeño A et al (2010) Yeast frataxin mutants display decreased superoxide dismutase activity crucial to promote protein oxidative damage. Free Radic Biol Med 48:411420. https://doi.org/10.1016/j.freeradbiomed.2009.11.010

45. Chandran V, Gao K, Swarup V et al (2017) Inducible and reversible phenotypes in a novel mouse model of Friedreich's Ataxia. Elife 6:e30054. https://doi.org/10.7554/eLife.30054

46. Puccio H, Simon D, Cossee M et al (2001) Mouse models for Friedreich ataxia exhibit cardiomyopathy, sensory nerve defect and Fe-S enzyme deficiency followed by intramitochondrial iron deposits. Nat Genet 27:181-186. https://doi.org/10.1038/84818

47. Ocana-Santero G, Díaz-Nido J, Herranz-Martín S (2021) Future Prospects of Gene Therapy for Friedreich's Ataxia. Int J Mol Sci 22:1815. https://doi.org/10.3390/ijms22041815

48. Perdomini M, Hick A, Puccio H, Pook MA (2013) Animal and cellular models of Friedreich ataxia. J Neurochem 126:65-79. https://doi.org/10.1111/jnc.12219

49. Fil D, Chacko BK, Conley R et al (2020) Mitochondrial damage and senescence phenotype of cells derived from a novel frataxin G127V point mutation mouse model of Friedreich's ataxia. Dis Model Mech. https://doi.org/10.1242/dmm.045229

Publisher's Note Springer Nature remains neutral with regard to jurisdictional claims in published maps and institutional affiliations. 